\title{
Taut contact hyperbolas on three-manifolds
}

\author{
Domenico Perrone ${ }^{1}$
}

Received: 7 February 2021 / Accepted: 1 July 2021 / Published online: 22 July 2021

(c) The Author(s) 2021

Dedicated to my grandson Davide and my grand-daughter Elisa

\begin{abstract}
In this paper, we introduce the notion of taut contact hyperbola on three-manifolds. It is the hyperbolic analogue of the taut contact circle notion introduced by Geiges and Gonzalo (Invent. Math., 121: 147-209, 1995), (J. Differ. Geom., 46: 236-286, 1997). Then, we characterize and study this notion, exhibiting several examples, and emphasizing differences and analogies between taut contact hyperbolas and taut contact circles. Moreover, we show that taut contact hyperbolas are related to some classic notions existing in the literature. In particular, it is related to the notion of conformally Anosov flow, to the critical point condition for the Chern-Hamilton energy functional and to the generalized Finsler structures introduced by R. Bryant. Moreover, taut contact hyperbolas are related to the bi-contact metric structures introduced in D. Perrone (Ann. Global Anal. Geom., 52: 213-235, 2017).
\end{abstract}

Keywords Taut contact hyperbolas - Taut contact circles · Bi-contact metric structures · Three-manifolds · 3D Lie groups · Generalized Finsler structures · Conformally Anosov flow $\cdot$ Chern-Hamilton energy functional

Mathematics Subject Classification $53 \mathrm{C} 15 \cdot 53 \mathrm{D} 10 \cdot 53 \mathrm{C} 25$

\section{Introduction}

Following $[13,15]$, a pair $\left(\eta_{1}, \eta_{2}\right)$ of contact 1 -forms on a three-manifold $M$ is called a contact circle if the linear combination $\eta_{a}=a_{1} \eta_{1}+a_{2} \eta_{2}$ is also a contact form for every $a=\left(a_{1}, a_{2}\right) \in \mathbb{S}^{1}$, the unit circle in $\mathbb{R}^{2}$. If in addition the volume forms $\eta_{a} \wedge\left(d \eta_{a}\right)$ are equal for every $a \in \mathbb{S}^{1}$, then $\left(\eta_{1}, \eta_{2}\right)$ is said to be a taut contact circle.

The author is member of INDAM-GNSAGA (Italy).

Domenico Perrone

domenico.perrone@unisalento.it

1 Dipartimento di Matematica e Fisica "E. De Giorgi”, Universitá del Salento, Via Provinciale Lecce-Arnesano, 73100 Lecce, Italy 
In the paper [24], we studied taut contact circles on a three-manifold from point of view of the Riemannian geometry and introduced the notion of bi-contact metric structure $\left(\eta_{1}, \eta_{2}, g\right)$, that is, $\left(\eta_{1}, \eta_{2}\right)$ is a pair of contact 1 -forms and $g$ is a Riemannian metric associated to both the contact forms $\eta_{1}, \eta_{2}$ such that the corresponding Reeb vector fields are orthogonal.

The main purpose of this paper is to start the study of the hyperbolic analogue, in dimension three, of taut contact circles study. The unit circle $\mathbb{S}^{1}$ of the Euclidean plane has its counterpart in the pseudo-Euclidean plane, that is, in the Minkowski plane, in the four arms of the unit equilateral hyperbolas $\mathbb{W}_{r}^{1}: x^{2}-y^{2}=r, r= \pm 1$. Then, we call contact hyperbola a pair of contact 1 -forms $\left(\eta_{1}, \eta_{2}\right)$ such that, for every $a=\left(a_{1}, a_{2}\right) \in \mathbb{H}_{r}^{1}$, the linear combination $\eta_{a}=a_{1} \eta_{1}+a_{2} \eta_{2}$ is also a contact form. If in addition the volume forms $\eta_{a} \wedge\left(d \eta_{a}\right)=r \eta_{1} \wedge\left(d \eta_{1}\right)$ for every $a \in \mathbb{M}_{r}^{1}$, then $\left(\eta_{1}, \eta_{2}\right)$ is said to be a taut contact hyperbola. In particular, if $\left(\eta_{1}, \eta_{2}, g\right)$ is a bi-contact metric structure on a three-manifold, then $\left(\eta_{1}, \eta_{2}\right)$ is either a taut contact circle or a taut contact hyperbola.

We note that the notion of taut contact hyperbola introduced in this paper is very natural because is related to some classic notions existing in the literature: the notion of conformally Anosov flow introduced by Mitsumatsu [21] and Eliashberg-Thurston [12], the critical point condition for the Chern-Hamilton energy functional $[9,26]$ and the generalized Finsler structures introduced by Bryant [5,6], are related to the taut contact hyperbolas. So we believe that this study is worthy of subsequent insights.

The present paper, where in particular we emphasize differences and analogies between taut contact hyperbolas and taut contact circles, is organized in the following way.

In Sect. 2, we collect some basic facts about contact Riemannian geometry.

In Sect. 3, we introduce the notion of taut contact hyperbola on a three-manifold. In particular, in the compact case, a taut contact hyperbola defines a conformally Anosov flow in the sense of Mitsumatsu [21] and Eliashberg-Thurston [12]. Then, we study left invariant taut contact hyperbolas on $3 D$ Lie groups. The Lie groups $\widetilde{S L}(2, R)$ and $\mathrm{Sol}^{3}$ are the only unimodular Lie groups which admit left invariant taut contact hyperbolas; thus, we determine the left invariant taut contact hyperbolas on these Lie groups. Then, we study taut contact hyperbolas on non-unimodular Lie groups, in particular there are non-unimodular Lie groups with the Milnor's invariant $\mathcal{D}=0$ which admit a taut contact hyperbola with the corresponding Reeb vector fields dependent.

Section 4 contains some characterization of taut contact hyperbolas (cf. Theorem 4.2), and a remark about a difference between a taut contact circle and a taut contact hyperbola in terms of symplectic structures.

In Sect. 5, in analogy with the notion of taut contact 2-sphere, we introduce the notion of taut contact 2-hyperboloid, in particular we get that the Lie group $\widetilde{S L}(2, R)$ is the only simply connected three-manifold which admits a taut contact 2-hyperboloid $\left(\eta_{1}, \eta_{2}, \eta_{3}\right)$ with the corresponding Reeb vector fields $\left(\xi_{1}, \xi_{2}, \xi_{3}\right)$ that constitute the frame dual of the coframe $\left(\eta_{1}, \eta_{2}, \eta_{3}\right)$.

In Sect. 6, we show that the critical point condition for the Chern-Hamilton energy functional $([9,26])$ is a sufficient condition for the existence of a taut contact hyperbola on a non-Sasakian contact metric three-manifold $(M, \eta, g)$ (cf. Theorem 6.1). In particular, in the compact case, $(M, \operatorname{ker} \eta)$ is universally tight. Thus, we exhibit an example related to this Theorem.

In Sect. 7 we study the geometry of a three-manifold $M$ determined by the existence of a bi-contact metric structure. We characterize the existence of a bi-contact metric structure $\left(\eta_{1}, \eta_{2}, g\right)$ on $M$ by the condition that $\left(\eta_{1}, \eta_{2}\right)$ is a $(-\varepsilon)$-Cartan structure (cf. Theorem 7.1). In particular, there are a 1 -form $\eta_{3}$ and a function $\kappa$, that we call the Webster function (cf. 
Remark 7.4), uniquely determined by this structure. Then, (cf. Theorem 7.5) $\eta_{3}$ is Killing (resp. a contact form) if and only if $\left(\eta_{1}, \eta_{2}\right)$ is taut contact circle (resp. the Webster function $\kappa \neq 0$ everywhere). Besides, we study the geometry of $M$ when the 1 -form $\eta_{3}$ is a contact form and in particular when the Webster function $\kappa= \pm 1$ (cf. Theorem 7.5 and Corollary 7.6).

Finally, in Sect. 8, we see how bi-contact metric structures are related to the generalized Finsler structures (introduced by Bryant $[5,6]$ ) and construct an explicit example of bi-contact metric structures $\left(\eta_{1}, \eta_{2}, g\right)$ where $\left(\eta_{1}, \eta_{2}\right)$ is a taut contact hyperbola with the Webster function $\kappa$ non-constant (in particular, this example gives a positive answer to a question posed in [24]).

\section{Riemannian geometry of contact manifolds}

In this section, we collect some basic facts about contact Riemannian geometry and refer to the two monographs $[3,4]$ for more information. All manifolds are supposed to be connected and smooth. Moreover, in what follows, for a Riemannian manifold $(M, g)$, we shall denote by $\nabla$ the Levi-Civita connection of the Riemannian metric $g$, by $R$ the corresponding Riemannian curvature tensor and by Ric the Ricci tensor.

A contact manifold is a $(2 n+1)$-dimensional manifold $M$ equipped with a global 1 -form $\eta$ such that $\eta \wedge(d \eta)^{n} \neq 0$ everywhere on $M$. It has an underlying almost contact structure $(\xi, \eta, \varphi)$ where $\xi$ is a global vector field (called the Reeb vector field, or the characteristic vector field) and $\varphi$ is a global tensor of type $(1,1)$ such that $\eta(\xi)=1, \varphi \xi=0, \varphi^{2}=-I+\eta \otimes \xi$. A Riemannian metric $g$ can be found such that

$$
\eta=g(\xi, \cdot), \quad d \eta=g(\cdot, \varphi \cdot) .
$$

In such a case, $g$ is called an associated metric, and we refer to $(M, \eta, g)$, or $(M, \xi, \eta, \varphi, g)$, as a contact metric (or contact Riemannian) manifold. The tensor $h=\frac{1}{2} \mathcal{L}_{\xi} \varphi$, where $\mathcal{L}$ denotes the Lie derivative, plays a fundamental role in contact Riemannian geometry, it is symmetric and satisfies: $h \varphi=-\varphi h, h \xi=0$ and

$$
\nabla \xi=-\varphi-\varphi h .
$$

In particular, the Reeb vector field $\xi$ is a geodesic vector field: $\nabla_{\xi} \xi=0$.

More in general, given an almost contact structure $(\xi, \eta, \varphi)$, a Riemannian metric $g$ can be found such that $g(\varphi X, \varphi Y)=g(X, Y)-\eta(X) \eta(Y)$, and in this case $(\xi, \eta, \varphi, g)$ is called almost contact metric structure. An almost contact structure $(\xi, \eta, \varphi)$ is said to be normal if the almost complex structure $J$ on $M \times \mathbb{R}$ defined by $J(X, f \mathrm{~d} / \mathrm{d} t)=(\phi X-f \xi, \eta(X) \mathrm{d} / \mathrm{d} t)$ is integrable, where $f$ is a real-valued function. A contact metric manifold is said to be a $K$-contact manifold if the Reeb vector field $\xi$ is a Killing vector field with respect to the associated metric $g$. Since the torsion $\tau=\mathcal{L}_{\xi} g$ satisfies $\tau=2 g(\cdot, h \varphi \cdot)$ and $\operatorname{Ric}(\xi, \xi)=2 n-t r h^{2}$, a contact metric manifold $M$ is $K$-contact if and only if the tensor $h=0$ or, equivalently, $\operatorname{Ric}(\xi, \xi)=2 n$. A contact metric manifold is said to be a Sasakian manifold if the almost contact structure $(\eta, \xi, \varphi)$ is normal. Any Sasakian manifold is $K$-contact and the converse also holds in dimension three. A contact metric manifold $(M, \eta, g)$ is said to be an $H$-contact manifold if Reeb vector field $\xi$ is a harmonic vector field, that is, $\xi$ satisfies the critical point condition for the energy functional defined on the space of all unit vector fields; moreover, a contact metric manifold $(M, \eta, g)$ is $H$-contact 
if and only if $\xi$ is an eigenvector of the Ricci operator $Q$, that is, $Q \xi=\left(2 n-\operatorname{tr} h^{2}\right) \xi$ [23]. Sasakian manifolds and $K$-contact manifolds are $H$-contact manifolds, but the converse, in general, is not true.

Recently, we have considered a Riemannian metric $g$ as an associated metric for two contact forms. More precisely, we have

Definition 2.1 ( [24]) Let $M$ be a three-manifold. A bi-contact metric structure on $M$ is a triple $\left(\eta_{1}, \eta_{2}, g\right)$ where $\left(\eta_{1}, \eta_{2}\right)$ is a pair of contact forms and $g$ is an associated metric for both the contact forms $\eta_{1}, \eta_{2}$, such that the corresponding Reeb vector fields satisfy $g\left(\xi_{1}, \xi_{2}\right)=0$, equivalently the corresponding almost contact structures $\left(\xi_{i}, \eta_{i}, \varphi_{i}\right), i=1,2$, satisfy the condition:

$$
\varphi_{1} \varphi_{2}+\varepsilon \eta_{1} \otimes \xi_{2}=-\left(\varphi_{2} \varphi_{1}+\varepsilon \eta_{2} \otimes \xi_{1}\right), \quad \varepsilon= \pm 1,
$$

where $\varepsilon$ is defined by $\varphi_{2} \xi_{1}=\varepsilon \varphi_{1} \xi_{2}$.

Then, in [24] we gave a complete classification of simply connected three-manifolds which admit a bi- $H$-contact metric structure $\left(\eta_{1}, \eta_{2}, g\right)$, i.e., $\left(\eta_{1}, g\right)$ and $\left(\eta_{2}, g\right)$ are both $H$-contact.

We note that in the classical definition of contact metric 3-structure (see, for example, [4] Chapter 13 and [3] Chapter 14) we have three contact metric structures $\left(\xi_{i}, \eta_{i}, \varphi_{i}, g\right)$, $i=1,2,3$, such that:

$$
\varphi_{i} \varphi_{j}-\eta_{j} \otimes \xi_{i}=\varphi_{k}=-\left(\varphi_{j} \varphi_{i}-\eta_{i} \otimes \xi_{j}\right)
$$

for any cyclic permutation $(i, j, k)$ of $(1,2,3)$. A contact metric 3-structure is called Sasakian 3-structure if the three contact metric structures are Sasakian. The condition (2.3) implies, in particular, the orthogonality of the three Reeb vector fields with respect to $g$. So, if $\left(\eta_{1}, \eta_{2}, \eta_{3}, g\right)$ is a contact metric 3 -structure then $\left(\eta_{i}, \eta_{j}, g\right)$ are bi-contact metric structures for any $i, j=1,2,3, i \neq j$. However, this is only a necessary condition for a contact metric 3-structure. In fact, the Lie group $\widetilde{S L}(2, \mathbb{R})$ admits three bi-contact metric structures which do not define a contact metric 3-structure (cf. Remark 7.7).

\section{Taut contact hyperbolas: first properties and examples}

\subsection{Definitions and first properties}

We begin this Subsection recalling the definitions of contact circle, contact sphere and taut contact circle introduced by $\mathrm{H}$. Geiges and J. Gonzalo on a manifold of dimension three (see, for example, $[13,15])$. In all this section, by $M$ we will denote always a three-manifold.

Let $\left(\eta_{1}, \eta_{2}\right)$ be a pair of contact 1 -forms on $M$. The pair $\left(\eta_{1}, \eta_{2}\right)$ is called a contact circle if for every $a=\left(a_{1}, a_{2}\right) \in \mathbb{S}^{1}$, the unit circle in $\mathbb{R}^{2}$, the linear combination $\eta_{a}=a_{1} \eta_{1}+a_{2} \eta_{2}$ is also a contact form. A contact circle $\left(\eta_{1}, \eta_{2}\right)$ is said to be a taut contact circle if the volume forms $\eta_{a} \wedge\left(d \eta_{a}\right)$ are equal for every $a \in \mathbb{S}^{1}$. Equivalently, a pair of contact forms $\left(\eta_{1}, \eta_{2}\right)$ is a taut contact circle if and only if

$$
\eta_{1} \wedge d \eta_{1}=\eta_{2} \wedge d \eta_{2} \quad \text { and } \quad \eta_{1} \wedge d \eta_{2}=-\eta_{2} \wedge d \eta_{1}
$$


In the case of closed three-manifolds, taut contact circles exist only on compact left quotients of the Lie groups: $\mathbb{S}^{3}=S U(2), \widetilde{S L}(2, R), \widetilde{E}(2)$ (cf. [13], Theorem 1.2 ).

The unit circle $\mathbb{S}^{1}$ of the Euclidean plane has its counterpart, in the pseudo-Euclidean plane, that is, in the Minkowski plane, in the four arms of the unit equilateral hyperbolas

$$
\mathbb{M}_{r}^{1}: x^{2}-y^{2}=r, r= \pm 1 .
$$

Indeed, the equilateral hyperbolas have many of the properties of circles in the Euclidean plane (cf., for example, [8]). So we give the following definitions.

Definition 3.1 A pair $\left(\eta_{1}, \eta_{2}\right)$ of contact forms on $M$ is called a contact hyperbola if for every $a=\left(a_{1}, a_{2}\right) \in \mathbb{T}_{r}^{1}$, the linear combination $\eta_{a}=a_{1} \eta_{1}+a_{2} \eta_{2}$ is also a contact form.

This definition implies that any non-trivial linear combination $\eta_{a}=a_{1} \eta_{1}+a_{2} \eta_{2}$ with constant coefficients $\left(a_{1}, a_{2}\right), a_{1}^{2}-a_{2}^{2} \neq 0$, is again a contact form.

Definition 3.2 A contact hyperbola $\left(\eta_{1}, \eta_{2}\right)$ is said to be a taut contact hyperbola if the volume forms $\eta_{a} \wedge\left(d \eta_{a}\right)$ satisfy

$$
\eta_{a} \wedge\left(d \eta_{a}\right)=r \eta_{1} \wedge\left(d \eta_{1}\right) \text { for all } a \in \mathbb{H}_{r}^{1} .
$$

(equivalently, $\left.\eta_{a} \wedge\left(d \eta_{a}\right)=-r \eta_{2} \wedge\left(d \eta_{2}\right)\right)$.

In [21], Mitsumatsu introduced a bi-contact structure $\left(\eta_{1}, \eta_{2}\right)$ on a three-manifold, that is, $\eta_{1}$ and $\eta_{2}$ are mutually transverse contact 1 -forms which induce opposite orientations. Anosov flow naturally induces a bi-contact structure whose intersection as a pair of plane fields is tangent to the flow. In general, the intersection of a bi-contact structure does not define an Anosov flow. In fact, he showed that if $\left(\eta_{1}, \eta_{2}\right)$ is a bi-contact structure on a compact three-manifold, then the vector field directing the intersection of the two contact subbundles is a conformally Anosov flow (that they called projectively Anosov flow) which is a generalization of an Anosov flow. Eliashberg and Thurston [12] studied bi-contact structures and conformally Anosov flows from the viewpoint of confoliation theory.

The next proposition shows that the notion of taut contact hyperbola is related to that of conformally Anosov flow.

Proposition 3.3 Let $\left(\eta_{1}, \eta_{2}\right)$ be a pair of contact forms on M. Then, $\left(\eta_{1}, \eta_{2}\right)$ is a taut contact hyperbola if and only if

$$
\eta_{2} \wedge d \eta_{2}=-\eta_{1} \wedge d \eta_{1} \quad \text { and } \quad \eta_{1} \wedge d \eta_{2}=-\eta_{2} \wedge d \eta_{1} .
$$

In particular, when $M$ is compact, a taut contact hyperbola defines a conformally Anosov flow. But, a pair of contact forms that defines a conformally Anosov flow, in general, does not define a taut contact hyperbola.

Proof Let $\left(\eta_{1}, \eta_{2}\right)$ be a taut contact hyperbola. If we take $a=(0,1) \in H_{r}^{1}, r=-1$, then $\eta_{a}=\eta_{2}$ and from (3.2) we get the condition $\eta_{2} \wedge d \eta_{2}=-\eta_{1} \wedge d \eta_{1}$. Moreover, for all $a \in \mathbb{H}_{r}^{1}$, $r= \pm 1,(3.2)$ and the above condition imply

$$
r \eta_{1} \wedge d \eta_{1}=\eta_{a} \wedge d \eta_{a}=\left(a_{1}^{2}-a_{2}^{2}\right) \eta_{1} \wedge d \eta_{1}+a_{1} a_{2}\left(\eta_{1} \wedge d \eta_{2}+\eta_{2} \wedge d \eta_{1}\right)
$$


and thus we get $\eta_{1} \wedge d \eta_{2}=-\eta_{2} \wedge d \eta_{1}$.

Vice versa, if we assume (3.3), from

$$
\eta_{a} \wedge d \eta_{a}=a_{1}^{2} \eta_{1} \wedge d \eta_{1}+a_{2}^{2} \eta_{2} \wedge d \eta_{2}+a_{1} a_{2}\left(\eta_{1} \wedge d \eta_{2}+\eta_{2} \wedge d \eta_{1}\right)
$$

we obtain (3.2). In particular, a taut contact hyperbola is a bi-contact structure (in the sense of Mitsumatsu) and thus, in the compact case, it defines a conformally Anosov flow. The last part is a consequence of Remark 3.10 .

Remark 3.4 By using notations of complex/hyperbolic numbers, the conditions (3.1) and (3.3) can be write, respectively, in the simple forms

$$
\eta^{c} \wedge d \eta^{c}=0, \text { where } \eta^{c}=\eta_{1}+i \eta_{2}, i^{2}=-1, \quad \eta^{h} \wedge d \eta^{h}=0, \text { where } \eta^{h}=\eta_{1}+j \eta_{2}, j^{2}=1, j \neq \pm 1 .
$$

Since the sphere $\mathbb{S}^{3}$ does not admit a conformally Anosov flow (cf. [21] , p.1420), from Proposition 3.3, follows that the sphere $\mathbb{S}^{3}$ does not admit a taut contact hyperbola (cf. also [24], Corollary 3.7).

Corollary 3.5 The torus $\mathbb{T}^{3}$ admits a taut contact hyperbola (and so a conformally Anosov flow).

Proof Consider on $\mathbb{R}^{3}$ the volume form $\Omega=d x \wedge d y \wedge d z$ and the contact 1-forms

$$
\eta_{1}=\cos z d x-\sin z d y \text { and } \eta_{2}=\cos z d x+\sin z d y .
$$

Then,

$$
\eta_{1} \wedge d \eta_{1}=\Omega=-\eta_{2} \wedge d \eta_{2} \quad \text { and } \quad \eta_{2} \wedge d \eta_{1}=\left(\cos ^{2} z-\sin ^{2} z\right) \Omega=-\eta_{1} \wedge d \eta_{2}
$$

Thus, by Proposition 3.3, $\left(\eta_{1}, \eta_{2}\right)$ defines a taut contact hyperbola on $\mathbb{R}^{3}$. On the other hand, $\eta_{1}$ and $\eta_{2}$ are invariant under translation by $2 \pi$; therefore, $\left(\eta_{1}, \eta_{2}\right)$ defines a taut contact hyperbola on the torus $\mathbb{T}^{3}$.

Remark 3.6 The torus $\mathbb{T}^{3}$ has many conformally Anosov flows, while it has no Anosov flows because its fundamental group does not grow exponentially.

Now, recall that a taut contact circle with $\eta_{1} \wedge d \eta_{2}=\eta_{2} \wedge d \eta_{1}=0$ is said to be a Cartan structure (see [13]). On the other hand, if (3.3) holds with $\eta_{1} \wedge d \eta_{2}=\eta_{2} \wedge d \eta_{1}=0$, we have a taut contact hyperbola like-Cartan structure. So it is natural to give the following

Definition 3.7 A pair of contact 1-forms $\left(\eta_{1}, \eta_{2}\right)$ is said to be a $(-\varepsilon)$-Cartan structure on the three-manifold $M$ if $\quad \eta_{2} \wedge d \eta_{2}=-\varepsilon \eta_{1} \wedge d \eta_{1} \quad$ and $\quad \eta_{1} \wedge d \eta_{2}=0=\eta_{2} \wedge d \eta_{1}, \varepsilon= \pm 1$.

Of course, a 1-Cartan structure is a Cartan structure and (-1)-Cartan structure is a taut contact hyperbola with $\eta_{1} \wedge d \eta_{2}=0$. 


\subsection{Taut contact hyperbolas on 3D Lie groups}

\subsection{Unimodular case}

Let $G$ be a simply connected unimodular $3 D$ Lie group. Then, $G$ contains a discrete subgroup $\Gamma$ such that the space of right cosets $\Gamma \backslash G$ is a differentiable manifold. Note that a three-dimensional Lie group $G$ admits a discrete subgroup $\Gamma$ such that $\Gamma \backslash G$ is compact if and only if $G$ is unimodular ( [20]). Moreover, each left-invariant tensor field on $G$ descends to $\Gamma \backslash G$.

In particular, if we have a left-invariant taut contact hyperbola on $G$, then it descends to $\Gamma \backslash G$ and thus defines a conformally Anosov flow.

Now, we determine the simply connected unimodular $3 D$ Lie groups $G$ which admit taut contact hyperbolas. Since $G$ is unimodular, there exist a basis of left invariant vector fields $\left(e_{1}, e_{2}, e_{3}\right)$ such that ( [20]):

$$
\left[e_{2}, e_{3}\right]=\lambda_{1} e_{1}, \quad\left[e_{3}, e_{1}\right]=\lambda_{2} e_{2}, \quad\left[e_{1}, e_{2}\right]=\lambda_{3} e_{3},
$$

where $\left(\lambda_{1}, \lambda_{2}, \lambda_{3}\right)$ are constant. If $\left(\lambda_{1}, \lambda_{2}, \lambda_{3}\right)=(0,0,0)$, then $G$ is the Abelian Lie group $\mathbb{R}^{3}$. The signature of $\left(\lambda_{1}, \lambda_{2}, \lambda_{3}\right) \neq(0,0,0)$ can be one of the following type:

- $\quad(+,+,+)$ and in this case $G$ is the three-sphere group $S U(2)$;

- $\quad(+,+, 0)$ and in this case $G=\widetilde{E}(2)$ (the universal cover of the group of orientationpreserving isometries of the Euclidean plane);

- $\quad(+, 0,0)$ and in this case $G$ is the Heisenberg group $\mathcal{H}^{3}=N i l^{3}$;

- $\quad(+,-,-)$ and in this case $G=\widetilde{S L}(2, R)$;

- $\quad(+,-, 0)$ and in this case $G=S^{3} l^{3}$ (also known as the group $E(1,1)$ of orientationpreserving isometries of the Minkowski plane).

Denote by $\eta_{i}$ the dual 1-forms : $\eta_{i}\left(e_{j}\right)=\delta_{i j}$. Then

$$
d \eta_{1}=-\lambda_{1} \eta_{2} \wedge \eta_{3}, \quad d \eta_{2}=-\lambda_{2} \eta_{3} \wedge \eta_{1}, \quad d \eta_{3}=-\lambda_{1} \eta_{1} \wedge \eta_{2},
$$

and thus

$$
\eta_{i} \wedge d \eta_{i}=-\lambda_{i} \eta_{1} \wedge \eta_{2} \wedge \eta_{3} \quad \text { and } \quad \eta_{i} \wedge d \eta_{j}=0 \quad \text { for any } i \neq j .
$$

In general, if $\left(\eta_{1}, \eta_{2}, \eta_{3}\right)$ is a coframe on a three-manifold, the non-trivial 1-forms $\eta_{a}=a_{1} \eta_{1}+a_{2} \eta_{2}+a_{3} \eta_{3}, \quad \eta_{b}=b_{1} \eta_{1}+b_{2} \eta_{2}+b_{3} \eta_{3}$ with constant coefficients $\left(a_{i}\right),\left(b_{i}\right)$, satisfy

$$
\begin{aligned}
\eta_{a} \wedge d \eta_{b}= & a_{1} b_{1} \eta_{1} \wedge d \eta_{1}+a_{2} b_{2} \eta_{2} \wedge d \eta_{2}+a_{3} b_{3} \eta_{3} \wedge d \eta_{3}+a_{1} b_{2} \eta_{1} \wedge d \eta_{2}+a_{2} b_{1} \eta_{2} \wedge d \eta_{1} \\
& +a_{1} b_{3} \eta_{1} \wedge d \eta_{3}+a_{3} b_{1} \eta_{3} \wedge d \eta_{1}+a_{2} b_{3} \eta_{2} \wedge d \eta_{3}+a_{3} b_{2} \eta_{3} \wedge d \eta_{2}
\end{aligned}
$$

Thus, two arbitrary left invariant 1 -forms $\eta_{a}, \eta_{b}$ on the unimodular Lie group $G$ satisfy

$$
\eta_{a} \wedge d \eta_{b}=-\mathcal{L}(a, b) \eta_{1} \wedge \eta_{2} \wedge \eta_{3}, \quad \text { where we put } \quad \mathcal{L}(a, b)=\lambda_{1} a_{1} b_{1}+\lambda_{2} a_{2} b_{2}+\lambda_{3} a_{3} b_{3} .
$$

Consequently, by Proposition 3.3, the left invariant 1 -forms $\left(\eta_{a}, \eta_{b}\right)$ define a taut contact hyperbola if and only if the symmetric bilinear map $\mathcal{L}$ satisfies 


$$
\mathcal{L}(a, a)=-\mathcal{L}(b, b) \neq 0, \quad \mathcal{L}(a, b)=0 .
$$

Therefore, the constant $\left(\lambda_{1}, \lambda_{2}, \lambda_{3}\right)$ that define the unimodular Lie groups $S U(2), \tilde{E}(2), \mathcal{H}^{3}$ and $\mathbb{R}^{3}$ do not satisfy the condition (3.6). Only the constant $\left(\lambda_{1}, \lambda_{2}, \lambda_{3}\right)$ that define the unimodular Lie groups $S o l^{3}$ and $\widetilde{S L}(2, \mathbb{R})$ satisfy the condition (3.6). Next, we determine the left invariant taut contact hyperbolas on these unimodular Lie groups.

Example 3.8 On the Lie group $\mathrm{Sol}^{3}$ we can consider a basis of left invariant vector fields $\left(e_{1}, e_{2}, e_{3}\right)$ such that

$$
\left[e_{2}, e_{3}\right]=2 e_{1}, \quad\left[e_{3}, e_{1}\right]=-2 e_{2}, \quad\left[e_{1}, e_{2}\right]=0 .
$$

In Example 8.2, we will give an explicit presentation of left invariant vector fields satisfying (3.7). The dual 1-forms $\eta_{i}$ satisfy

$$
d \eta_{1}=-2 \eta_{2} \wedge \eta_{3}, \quad d \eta_{2}=2 \eta_{3} \wedge \eta_{1}, \quad d \eta_{3}=0,
$$

and thus

$$
\eta_{1} \wedge d \eta_{1}=-2 \eta_{1} \wedge \eta_{2} \wedge \eta_{3}=-\eta_{2} \wedge d \eta_{2}, \quad \eta_{1} \wedge d \eta_{2}=0=\eta_{2} \wedge d \eta_{1} .
$$

Therefore, by $(3.6)\left(\eta_{1}, \eta_{2}\right)$ is a left invariant taut contact hyperbola on the unimodular Lie group $\mathrm{Sol}^{3}$ and this structure descends to any compact left-quotient.

Moreover, by using (3.6), two arbitrary left invariant 1 -forms $\left(\eta_{a}, \eta_{b}\right)$ on the Lie group $\mathrm{Sol}^{3}$ define a taut contact hyperbola if and only if

$$
\left(a_{1}^{2}-a_{2}^{2}\right) \neq 0 \text { and }\left(b_{1}, b_{2}\right)= \pm\left(a_{2}, a_{1}\right) .
$$

Example 3.9 On the Lie group $\widetilde{S L}(2, \mathbb{R})$, we can consider a basis of left invariant vector fields $\left(e_{1}, e_{2}, e_{3}\right)$ such that

$$
\left[e_{2}, e_{3}\right]=2 e_{1}, \quad\left[e_{3}, e_{1}\right]=-2 e_{2}, \quad\left[e_{1}, e_{2}\right]=-2 e_{3} .
$$

In Example 8.2, we will give an explicit presentation of left invariant vector fields satisfying (3.8). The dual 1-forms $\eta_{i}$ satisfy

$$
d \eta_{1}=-2 \eta_{2} \wedge \eta_{3}, \quad d \eta_{2}=2 \eta_{3} \wedge \eta_{1}, \quad d \eta_{3}=2 \eta_{1} \wedge \eta_{2},
$$

and thus

$$
-\eta_{1} \wedge d \eta_{1}=2 \eta_{1} \wedge \eta_{2} \wedge \eta_{3}=\eta_{2} \wedge d \eta_{2}=\eta_{3} \wedge d \eta_{3}, \quad \eta_{1} \wedge d \eta_{j}=0 \text { for any } i \neq j
$$

Therefore, $\left(\eta_{1}, \eta_{2}\right)$ and $\left(\eta_{1}, \eta_{3}\right)$ satisfy (3.6), i.e., they are left invariant taut contact hyperbolas on the unimodular Lie group $\widetilde{S L}(2, \mathbb{R})$ and these structures descend to any compact left-quotient.

Moreover, we can classify all left invariant taut contact hyperbolas on the Lie group $\widetilde{S L}(2, \mathbb{R})$. In this case, the bilinear map $\mathcal{L}$ defines on $\mathbb{R}^{3}$ the Lorentzian metric

$$
g_{0}(a, b)=\mathcal{L}(a, b)=a_{1} b_{1}-a_{2} b_{2}-a_{3} b_{3}, \text { for any } a, b \in \mathbb{R}^{3} .
$$

Then, by (3.6), two arbitrary left invariant contact 1 -forms $\eta_{a}, \eta_{b}$ on $\widetilde{S L}(2, \mathbb{R})$, define a taut contact hyperbola if and only if $g_{0}(b, b)=-g_{0}(a, a) \neq 0$ and $g_{0}(a, b)=0$, i.e., the vectors $a$ and $b$ are two orthogonal vectors, one of which is timelike and so the other is spacelike. 
Remark 3.10 We can consider two left invariant 1-forms $\eta_{a}, \eta_{b}$ on the Lie group $S o l^{3}$ with

$$
\mathcal{L}(b, b)=b_{1}^{2}-b_{2}^{2}=-\left(a_{1}^{2}-a_{2}^{2}\right)=-\mathcal{L}(a, a) \neq 0 \text { and } \mathcal{L}(a, b)=a_{1} b_{1}-a_{2} b_{2} \neq 0,
$$

then $\eta_{a}, \eta_{b}$ satisfy $\eta_{b} \wedge d \eta_{b}=-\eta_{a} \wedge d \eta_{a}$ and $\eta_{a} \wedge d \eta_{b}=\eta_{b} \wedge d \eta_{a} \neq 0$. Analogously, we can consider $\eta_{a}^{\prime}, \eta_{b}^{\prime}$ on the Lie group $S L(2, \mathbb{R})$ with

$$
g_{0}(b, b)=-g_{0}(a, a) \neq 0 \text { and } g_{0}(a, b) \neq 0 .
$$

In both the cases, $\left(\eta_{a}, \eta_{b}\right)$ and $\left(\eta_{a}^{\prime}, \eta_{b}^{\prime}\right)$ do not define a taut contact hyperbola, but they define a bi-contact structure in the sense of Mitsumatsu [21], and so define a conformally Anosov on any compact quotient of $S_{0} l^{3}$ and $\widetilde{S L}(2, \mathbb{R})$, respectively. Therefore, the notion of taut contact hyperbola is stronger than the notion of conformally Anosov.

\subsection{Non-unimodular case}

Let $G$ be a non-unimodular $3 D$ Lie group. Then $G$ admits a basis of left invariant vector fields $e_{1}, e_{2}, e_{3}$ such that (cf. [20])

$$
\left[e_{1}, e_{2}\right]=\alpha e_{2}+\beta e_{3}, \quad\left[e_{1}, e_{3}\right]=\gamma e_{2}+\delta e_{3}, \quad\left[e_{2}, e_{3}\right]=0, \quad \alpha+\delta \neq 0 .
$$

This Lie group $G$ can be presented as a semi-direct product Lie group $\mathbb{R}^{2} \rtimes_{A} \mathbb{R}$, where $A=\left(\begin{array}{ll}\alpha & \gamma \\ \beta & \delta\end{array}\right), \operatorname{tr} A=\alpha+\delta \neq 0$. Denote by

$$
\mathcal{D}=4 \operatorname{det} A /(\operatorname{tr} A)^{2}
$$

the invariant introduced by Milnor [20, p. 321], which, unless $A$ is a multiple of the identity matrix, completely determines the non-unimodular Lie algebra (and so the Lie group) up to isomorphisms.

Let $\left(\vartheta^{1}, \vartheta^{2}, \vartheta^{3}\right)$ be the basis of 1 -forms dual of $\left(e_{1}, e_{2}, e_{3}\right)$. Then,

$$
d \vartheta^{1}=0, \quad d \vartheta^{2}=-\alpha \vartheta^{1} \wedge \vartheta^{2}-\gamma \vartheta^{1} \wedge \vartheta^{3}, \quad d \vartheta^{3}=-\beta \vartheta^{1} \wedge \vartheta^{2}-\delta \vartheta^{1} \wedge \vartheta^{3},
$$

and thus

$$
\vartheta^{2} \wedge d \vartheta^{2}=\gamma \Omega, \quad \vartheta^{3} \wedge d \vartheta^{3}=-\beta \Omega, \quad \vartheta^{2} \wedge d \vartheta^{3}=\delta \Omega \quad \text { and } \quad \vartheta^{3} \wedge d \vartheta^{2}=-\alpha \Omega,
$$

where $\Omega=\vartheta^{1} \wedge \vartheta^{2} \wedge \vartheta^{3}$. Then, by using (3.5), two arbitrary left invariant 1-forms $\eta_{a}=\sum_{i=1}^{3} a_{i} \vartheta^{i}$ and $\eta_{b}=\sum_{i=1}^{3} b_{i} \vartheta^{i}$ satisfy

$$
\begin{aligned}
& \eta_{a} \wedge d \eta_{b}=\left(\gamma a_{2} b_{2}+\delta a_{2} b_{3}-\alpha a_{3} b_{2}-\beta a_{3} b_{3}\right) \Omega, \\
& \eta_{a} \wedge d \eta_{a}=\left(\gamma a_{2}^{2}+(\delta-\alpha) a_{2} a_{3}-\beta a_{3}^{3}\right) \Omega .
\end{aligned}
$$

Therefore, $\left(\eta_{a}, \eta_{b}\right)$ is a taut contact hyperbola on the non-unimodular Lie group $G$ if, and only if, are satisfied the following:

$$
\left\{\begin{array}{c}
\left(\gamma a_{2}^{2}+(\delta-\alpha) a_{2} a_{3}-\beta a_{3}^{2}\right)=-\left(\gamma b_{2}^{2}+(\delta-\alpha) b_{2} b_{3}-\beta b_{3}^{2}\right) \neq 0 \\
2 \gamma a_{2} b_{2}+(\delta-\alpha)\left(a_{2} b_{3}+a_{3} b_{2}\right)-2 \beta a_{3} b_{3}=0 .
\end{array}\right.
$$

In particular, 


$$
\left(\vartheta^{2}, \vartheta^{3}\right) \text { is a taut contact hyperbola } \Longleftrightarrow \beta=\gamma \neq 0 \text { and } \alpha=\delta \neq 0 \text {. }
$$

In this case, the non-unimodular Lie group is defined by the matrix $A=\left(\begin{array}{cc}\alpha & \beta \\ \beta & \alpha\end{array}\right), \alpha, \beta \neq 0$, and the Reeb vector fields of $\vartheta^{2}, \vartheta^{3}$ are given by $\xi_{2}=e_{2}-(\alpha / \beta) e_{3}, \quad \xi_{3}=-(\alpha / \beta) e_{2}+e_{3}$. Then, $\xi_{2}, \xi_{3}$ are linearly independent if and only if the Milnor's invariant $\mathcal{D}=4 \operatorname{det} A /(\operatorname{tr} A)^{2} \neq 0$. Moreover, $\mathcal{D}=0$ if and only if $\xi_{2}= \pm \xi_{3}$. Thus, we get the following

Proposition 3.11 The non-unimodular Lie group $G=\mathbb{R}^{2} \rtimes_{A} \mathbb{R}$, where $A=\left(\begin{array}{cc}\alpha & \pm \alpha \\ \pm \alpha & \alpha\end{array}\right)$, $\alpha \neq 0$, admits a taut contact hyperbola $\left(\eta_{1}, \eta_{2}\right)$ with the corresponding Reeb vector fields satisfying $\xi_{2}=\mp \xi_{1}$.

Remark 3.12 The result of the Proposition 3.11 gives an interesting difference with respect to the case of a taut contact circle. In fact, the Reeb vector fields of any taut contact circle are linearly independent (cf. Theorem 4.1).

Remark 3.13 Not all non-unimodular Lie groups admit a left invariant taut contact hyperbola. In fact, for $\alpha=\delta \neq 0$ and $\beta \gamma<0$, the system (3.10) does not admit solution.

Now, we give an explicit example of non-unimodular Lie group satisfying Proposition 3.11 .

Example 3.14 Consider the hyperbolic plane $\mathbb{H}^{2}=\left\{\left(x_{1}, x_{2}\right) \in \mathbb{R}^{2}: x_{2}>0\right\}$ equipped with standard Lie group structure. For any $\alpha \neq 0$, the vector fields

$$
E_{1}=2 \alpha x_{2} \partial_{1}, \quad E_{2}=2 \alpha x_{2} \partial_{2}
$$

define a basis of left invariant vector fields on $\mathbb{\boxplus}^{2}$. Now, consider the direct product Lie group

$$
\mathcal{G}_{\mathcal{H}}=\mathbb{H}^{2} \times \mathbb{R} .
$$

Then $\left(E_{1}, E_{2}, E_{3}=\partial_{t}\right)$ is a basis of left invariant vector fields on $\mathcal{G}_{\mathcal{H}}$. We note that with respect to the basis

$$
e_{1}=E_{2}, \quad e_{2}=\left(E_{1}+E_{3}\right), \quad e_{3}=\left(E_{1}-E_{3}\right),
$$

the Lie algebra of $\mathcal{G}_{\mathcal{H}}$ is defined by $\left[e_{2}, e_{3}\right]=0, \quad\left[e_{1}, e_{2}\right]=\left[e_{1}, e_{3}\right]=\alpha\left(e_{2}+e_{3}\right)$. So, $\mathcal{G}_{\mathcal{H}}$ is the non-unimodular Lie group $\mathbb{R}^{2} \rtimes_{A} \mathbb{R}$, where $A=\alpha I_{2}$, with the Milnor's invariant $\mathcal{D}=0$.

\section{Some characterization of taut contact hyperbolas}

We start this section recalling the following characterizations of taut contact circles. 
Theorem 4.1 (Zessin [27]) Let $\left(\eta_{1}, \eta_{2}\right)$ be a contact circle on a three-manifold $M$, and let $\xi_{1}$, $\xi_{2}$ be the corresponding Reeb vector fields. Then, $\xi_{1}, \xi_{2}$ are everywhere linearly independent and $d \eta_{1}\left(\xi_{2}, \cdot\right), d \eta_{2}\left(\xi_{1}, \cdot\right)$ never vanish. Moreover, the following properties are equivalent

(i) $\quad\left(\eta_{1}, \eta_{2}\right)$ is a taut contact circle;

(ii) $\xi_{a}=a_{1} \xi_{1}+a_{2} \xi_{2}$ is the Reeb vector field of $\eta_{a}=a_{1} \eta_{1}+a_{2} \eta_{2}$ for any $a \in \mathbb{S}^{1}$;

(iii) $\eta_{2}\left(\xi_{1}\right)=-\eta_{1}\left(\xi_{2}\right)$ and $d \eta_{1}\left(\xi_{2}, \cdot\right)=-d \eta_{2}\left(\xi_{1}, \cdot\right)$.

In Proposition 3.11, we showed the existence of taut contact hyperbolas $\left(\eta_{1}, \eta_{2}\right)$ with the corresponding Reeb vector fields $\left(\xi_{1}, \xi_{2}= \pm \xi_{1}\right)$. Next, we give some characterization of taut contact hyperbolas with $\xi_{2} \neq \pm \xi_{1}$. More precisely, we show the following theorem.

Theorem 4.2 Let $\left(\eta_{1}, \eta_{2}\right)$ be a pair of contact forms on a three-manifold $M$ with $\xi_{2} \neq \pm \xi_{1}$ in any point $p \in M$. Then, the following are equivalent:

(a) $\left(\eta_{1}, \eta_{2}\right)$ is a taut contact hyperbola ;

(b) $\eta_{1}\left(\xi_{2}\right)=\eta_{2}\left(\xi_{1}\right)$ and $d \eta_{1}\left(\xi_{2}, \cdot\right)=d \eta_{2}\left(\xi_{1}, \cdot\right) \neq 0$ in any point $p \in M$;

(c) $\eta_{a}=a_{1} \eta_{1}+a_{2} \eta_{2}$ is a contact form with Reeb vector field $\xi_{a}=r\left(a_{1} \xi_{1}-a_{2} \xi_{2}\right)$ for any $a \in \mathbb{H}_{r}^{1}$.

In particular, in a such case, $\xi_{1}, \xi_{2}$ are linearly independent.

We first give the following lemmas.

Lemma 4.3 Let $\left(\eta_{1}, \eta_{2}\right)$ be a contact hyperbola on $M$. Then,

$\xi_{1}, \xi_{2}$ are pointwise linearly independent $\Longleftrightarrow d \eta_{1}\left(\xi_{2}, \cdot\right)$ and $d \eta_{2}\left(\xi_{1}, \cdot\right)$ are $\neq 0$ in any point.

Moreover,

$\xi_{a}=r\left(a_{1} \xi_{1}-a_{2} \xi_{2}\right)$ is the Reeb vector field of $\eta_{a}=a_{1} \eta_{1}+a_{2} \eta_{2}$ for all $a \in \mathbb{M}_{r}^{1}$ if and only if $\eta_{2}\left(\xi_{1}\right)=\eta_{1}\left(\xi_{2}\right)$ and $d \eta_{1}\left(\xi_{2}, \cdot\right)=d \eta_{2}\left(\xi_{1}, \cdot\right)$.

Proof $(\Rightarrow)$ Suppose that there exists a point $p \in M$ such that $d \eta_{2}\left(\xi_{1}, \cdot\right)=0$ at $p$, and so $d \eta_{a}\left(\xi_{1}, \cdot\right)=a_{1} d \eta_{1}\left(\xi_{1}, \cdot\right)+a_{2} d \eta_{2}\left(\xi_{1}, \cdot\right)=0$ at $p$ for any $a \in \mathbb{T}_{r}^{1}$. Then, since $\left(\eta_{a} \wedge d \eta_{a}\right)\left(\xi_{1}, \cdot, \cdot\right) \neq 0$, we get $\eta_{a}\left(\xi_{1}\right)_{p} \neq 0$ for any $a \in \mathbb{H}_{r}^{1}$, and in particular $\eta_{2}\left(\xi_{1}\right)_{p} \neq 0$. Now, we put $\xi_{1}^{\prime}=(1 / \varrho) \xi_{1}$, where $\varrho=\eta_{2}\left(\xi_{1}\right)_{p} \neq 0$. Then, $\eta_{2}\left(\xi_{1}^{\prime}\right)_{p}=1$ and $\left(d \eta_{2}\right)\left(\xi_{1}^{\prime}, \cdot\right)=0$ give the contradiction $\left(\xi_{2}\right)_{p}=(1 / \varrho)\left(\xi_{1}\right)_{p}$, that is, $\xi_{1}, \xi_{2}$ linearly dependent at $p$.

$(\Leftarrow)$ If we suppose $\xi_{1}, \xi_{2}$ linearly dependent in some point $p$, i.e., $\left(\xi_{2}\right)_{p}=\rho\left(\xi_{1}\right)_{p}$ for some constant $\varrho \neq 0$, then we have the contradiction $\left(d \eta_{1}\right)\left(\xi_{2}, \cdot\right)_{p}=\rho\left(d \eta_{1}\right)\left(\xi_{1}, \cdot\right)_{p}=0$. For the second part, it is sufficient to remark that

$$
\eta_{a}\left(\xi_{a}\right)=1+r a_{1} a_{2}\left(\eta_{2}\left(\xi_{1}\right)-\eta_{1}\left(\xi_{2}\right)\right) \text { and } d \eta_{a}\left(\xi_{a}, \cdot\right)=r a_{1} a_{2}\left(d \eta_{2}\left(\xi_{1}, \cdot\right)-d \eta_{1}\left(\xi_{2}, \cdot\right)\right) \text {. }
$$


Lemma 4.4 Let $\left(\eta_{1}, \eta_{2}\right)$ be a contact hyperbola on $M$ with $\xi_{2} \neq \pm \xi_{1}$ in any point $p \in M$. Then, $d \eta_{1}\left(\xi_{2}, \cdot\right)$ and $d \eta_{2}\left(\xi_{1}, \cdot\right)$ are $\neq 0$ in any point $p \in M$.

In particular, if $\xi_{2} \neq \pm \xi_{1}$ in any point $p \in M$, then $\xi_{1}, \xi_{2}$ are linearly independent in any point $p \in M$.

Proof Assume that $\xi_{2} \neq \pm \xi_{1}$ in any point, and suppose that there exists a point $p \in M$ such that $d \eta_{2}\left(\xi_{1}, \cdot\right)_{p}=0$. Then, for all $a \in \mathbb{T}_{r}^{1}$

$$
d \eta_{a}\left(\xi_{1}, \cdot\right)=a_{1} d \eta_{1}\left(\xi_{1}, \cdot\right)+a_{2} d \eta_{2}\left(\xi_{1}, \cdot\right)=0 \text { at } p .
$$

Consequently, since $\eta_{a}$ is a contact form, i.e., $\eta_{a} \wedge d \eta_{a}\left(\xi_{1}, \cdot, \cdot\right) \neq 0$, we obtain

$$
\eta_{a}\left(\xi_{1}\right)_{p} \neq 0 \text { for all } a \in \mathbb{H}_{r}^{1} \text {. }
$$

In particular, taking $a=(0,1)$, we have that $\lambda:=\eta_{2}\left(\xi_{1}\right)_{p} \neq 0$.

Now, we show that $\lambda \neq 0$ gives a contradiction. Consider the function $f: \mathbb{H}_{r}^{1} \rightarrow \mathbb{R}$ defined by

$$
f(a)=\eta_{a}\left(\xi_{1}\right)_{p}=a_{1} \eta_{1}\left(\xi_{1}\right)_{p}+a_{2} \eta_{2}\left(\xi_{1}\right)_{p}=a_{1}+\lambda a_{2} .
$$

Consider the cases: (I) $\lambda>0$ and (II) $\lambda<0$.

For the case $(I)$, we distinguish the following subcases:

$$
\left(I_{1}\right) \lambda>1, \quad\left(I_{2}\right) 0<\lambda<1, \quad\left(I_{3}\right) \lambda=1 .
$$

For the subcase $\left(I_{1}\right)$ consider the function $f$ defined on the connected subset $C_{1}=\left\{a \in \mathbb{R}^{2}: a_{1}^{2}-a_{2}^{2}=1, a_{1}>0\right\}$. Put $\mu=\left(\lambda / \sqrt{\lambda^{2}-1}\right)>1$ and take

$$
a=\left(a_{1}, a_{2}\right), \bar{a}=\left(a_{1},-a_{2}\right) \in C_{1} \text {, with } a_{1}>\mu>1 \text { and } a_{2}=\sqrt{a_{1}^{2}-1}>0 .
$$

Then, $f(a)=a_{1}+\lambda a_{2}>0$ and $f(\bar{a})=a_{1}-\lambda a_{2}<0$. Thus, it should exist $b \in C_{1}$ such that $f(b)=0$, and this gives a contradiction.

For the subcase $\left(I_{2}\right)$ consider the function $f$ defined on the connected subset $C_{2}=\left\{a \in \mathbb{R}^{2}: a_{1}^{2}-a_{2}^{2}=-1, a_{2}>0\right\}$. Put $\mu=(1 / \lambda)>1$ and take

$$
a=\left(a_{1}, a_{2}\right), \bar{a}=\left(-a_{1}, a_{2}\right) \in C_{2} \text {, with } a_{1}>0 \text { and } a_{2}>\mu / \sqrt{\mu^{2}-1}>0 .
$$

Then, $f(a)=a_{1}+\lambda a_{2}>0$ and $f(\bar{a})=-a_{1}+\lambda a_{2}<0$. Thus, it should exist $b \in C_{2}$ such that $f(b)=0$, and this gives a contradiction.

For the subcase $\left(I_{3}\right), \eta_{2}\left(\xi_{1}\right)_{p}=1$ and $d \eta_{2}\left(\xi_{1}, \cdot\right)_{p}=0$ give the contradiction $\xi_{2}=\xi_{1}$ at $p$.

For the case $(I I)$, we distinguish the following subcases:

$$
\left(I I_{1}\right) \lambda<-1, \quad\left(I I_{2}\right)-1<\lambda<0, \quad\left(I_{3}\right) \lambda=-1 .
$$

For the subcase $\left(I I_{1}\right)$ consider the function $f$ defined on the connected subset $C_{3}=\left\{a \in \mathbb{R}^{2}: a_{1}^{2}-a_{2}^{2}=1, a_{1}<0\right\}$. Put $\mu=\left(\lambda / \sqrt{\lambda^{2}-1}\right)<-1$ and take

$$
a=\left(a_{1}, a_{2}\right), \bar{a}=\left(a_{1},-a_{2}\right) \in C_{3} \text {, with } a_{1}<\mu<-1 \text { and } a_{2}=\sqrt{a_{1}^{2}-1}>0 .
$$

Then, $f(a)=a_{1}+\lambda a_{2}<0$ and $f(\bar{a})=a_{1}-\lambda a_{2}>0$. Thus, it should exist $b \in C_{3}$ such that $f(b)=0$, and this gives a contradiction. 
For the subcase $\left(I I_{2}\right)$ consider the function $f$ defined on the connected subset $C_{4}=\left\{a \in \mathbb{R}^{2}: a_{1}^{2}-a_{2}^{2}=-1, a_{2}<0\right\}$. Put $\mu=(1 / \lambda)<-1$ and take

$$
a=\left(a_{1}, a_{2}\right), \bar{a}=\left(-a_{1}, a_{2}\right) \in C_{4} \text {, with } a_{1}=\sqrt{a_{2}^{2}-1}>0 \text { and } a_{2}<\mu / \sqrt{\mu^{2}-1}<-1 .
$$

Then, $f(a)=a_{1}+\lambda a_{2}>0$ and $f(\bar{a})=-a_{1}+\lambda a_{2}<0$. Thus, it should exist $b \in C_{4}$ such that $f(b)=0$, and this gives a contradiction.

For the subcase $\left(I I_{3}\right), \eta_{2}\left(-\xi_{1}\right)_{p}=1$ and $d \eta_{2}\left(-\xi_{1}, \cdot\right)_{p}=0$ give the contradiction $\xi_{2}=-\xi_{1}$ at $p$. We proceed analogously if suppose $d \eta_{1}\left(\xi_{2}, \cdot\right)_{p}=0$. The second part of the Lemma follows from Lemma 4.3.

Proof of Theorem $4.2(a) \Longleftrightarrow(b)$

If we suppose (a) (respectively (b)), by Lemma 4.4 (respectively Lemma 4.3), we have that the Reeb vector fields $\xi_{1}, \xi_{2}$ are linearly independent. Moreover, we have

$$
\begin{aligned}
& \eta_{1} \wedge d \eta_{1}\left(\xi_{1}, \xi_{2}, \cdot\right)=d \eta_{1}\left(\xi_{2}, \cdot\right), \quad \eta_{2} \wedge d \eta_{2}\left(\xi_{1}, \xi_{2}, \cdot\right)=-d \eta_{2}\left(\xi_{1}, \cdot\right), \\
& \eta_{1} \wedge d \eta_{2}\left(\xi_{1}, \xi_{2}, \cdot\right)=-\eta_{1}\left(\xi_{2}\right) d \eta_{2}\left(\xi_{1}, \cdot\right), \quad \eta_{2} \wedge d \eta_{1}\left(\xi_{1}, \xi_{2}, \cdot\right)=\eta_{2}\left(\xi_{1}\right) d \eta_{1}\left(\xi_{2}, \cdot\right) .
\end{aligned}
$$

Then, by using Proposition 3.3, it is not difficult to see that $(a) \Leftrightarrow(b)$.

$(c) \Longleftrightarrow(a)$

Suppose $(c)$, that is, $\eta_{a}=a_{1} \eta_{1}+a_{2} \eta_{2}$ is a contact form with Reeb vector field $\xi_{a}=r\left(a_{1} \xi_{1}-a_{2} \xi_{2}\right)$ for any $a \in \mathbb{H}_{r}^{1}$. Then, $\left(\eta_{1}, \eta_{2}\right)$ is a contact hyperbola and, from Lemma $4.4, \xi_{1}, \xi_{2}$ are linearly independent and $d \eta_{1}\left(\xi_{2}, \cdot\right), d \eta_{2}\left(\xi_{1}, \cdot\right) \neq 0$ in any point $p \in M$. Then, Lemma 4.3 gives $\eta_{1}\left(\xi_{2}\right)=\eta_{2}\left(\xi_{1}\right)$ and $d \eta_{1}\left(\xi_{2}, \cdot\right)=d \eta_{2}\left(\xi_{1}, \cdot\right) \neq 0$. So, we get $(b)$ and thus $(a)$.

Conversely, suppose $(a)$, that is, $\left(\eta_{1}, \eta_{2}\right)$ is a taut contact hyperbola and thus $\eta_{a}=a_{1} \eta_{1}+a_{2} \eta_{2}$ is a contact form for any $a \in \mathbb{G}_{r}^{1}$. Since $(a)$ is equivalent to $(b)$, by Lemma 4.3 we get $(c)$.

We close this subsection with remarking that the taut contact hyperbolas are related to the symplectic pair.

\section{Remark 4.5 (taut contact hyperbola/circle and symplectic structures)}

Let $(M, \eta, \xi, \varphi)$ be an almost contact manifold. We denote by $C(M)=\mathbb{R}_{+} \times M$ the cone on $M$, for more information about the geometry of the cone $C(M)$ we refer, for example, to [4] Section 6.5. Consider the $(1,1)$-tensor $J$ on $C(M)$ defined by

$$
J X=\varphi X \text { for } X \in \operatorname{ker} \eta, \quad J \xi=\zeta, \quad J \zeta=-\xi,
$$

where $\zeta=t \frac{\partial}{\partial t}$ is Liouville (or Euler) vector field. Then, $J$ is an almost complex structure invariant under the flow of $\zeta: \mathcal{L}_{\zeta} J=0$. Moreover, it well-known that $\eta$ is a contact form on $M$ if and only if the 2-form $\Omega=d\left(t^{2} \eta\right)$ is a symplectic form on $C(M)$.

Now, let $\left(\eta_{1}, \eta_{2}\right)$ be a pair of contact forms on a three-manifold $M$ and $\left(\eta_{i}, \xi_{i}, \varphi_{i}\right)$, $i=1,2$, underlying almost contact structures. Then the corresponding symplectic forms $\Omega_{i}=d\left(t^{2} \eta_{i}\right)=2 t d t \wedge \eta_{i}+t^{2} d \eta_{i}, i=1,2$, satisfy:

$$
\begin{aligned}
& \Omega_{1} \wedge \Omega_{1}==4 t^{3} d t \wedge \eta_{1} \wedge d \eta_{1}, \\
& \Omega_{2} \wedge \Omega_{2}=4 t^{3} d t \wedge \eta_{2} \wedge d \eta_{2}, \\
& \Omega_{1} \wedge \Omega_{2}=2 t^{3} d t \wedge\left(\eta_{1} \wedge d \eta_{2}+\eta_{2} \wedge d \eta_{1}\right)=\Omega_{2} \wedge \Omega_{1}, \\
&\left(a_{1} \Omega_{1}+a_{2} \Omega_{2}\right) \wedge\left(a_{1} \Omega_{1}+a_{2} \Omega_{2}\right)=4 t^{3} d t \wedge \eta_{a} \wedge d \eta_{a} .
\end{aligned}
$$


So, $\left(\eta_{1}, \eta_{2}\right)$ is a contact hyperbola (resp. circle) if and only if $\Omega_{a}:=\left(a_{1} \Omega_{1}+a_{2} \Omega_{2}\right)$ is a symplectic 2-form on the four-dimensional cone $C(M)$ for any $\left(a_{1}, a_{2}\right) \in \mathbb{H}_{r}^{1}$ (resp. for any $\left.\left(a_{1}, a_{2}\right) \in \mathbb{S}^{1}\right)$.

On the other hand, on a four-manifold, following Bande and Kotschick [2]: a symplectic pair is defined by two symplectic forms $\left(\omega_{1}, \omega_{2}\right)$ that satisfy

$$
\omega_{1} \wedge \omega_{2}=0 \text { and } \omega_{1} \wedge \omega_{1}=-\omega_{2} \wedge \omega_{2},
$$

and following Geiges [14]: $\left(\omega_{1}, \omega_{2}\right)$ is said to be a conformal symplectic couple if

$$
\omega_{1} \wedge \omega_{2}=0 \text { and } \omega_{1} \wedge \omega_{1}=\omega_{2} \wedge \omega_{2} .
$$

Therefore (cf. also Section 6 of [24] where we studied the metric cone of a bi-contact metric manifold) we get:

$\left(\eta_{1}, \eta_{2}\right)$ is a taut contact hyperbola (resp. circle) if and only if the corresponding symplectic 2-forms $\left(\Omega_{1}, \Omega_{2}\right)$ define a symplectic pair (resp. a conformal symplectic couple) .

Moreover, given a pair of contact forms $\left(\eta_{1}, \eta_{2}\right)$ on $M$ with $\xi_{2} \neq \pm \xi_{1}$ in any point $p \in M$, the corresponding symplectic 2-forms $\Omega_{1}, \Omega_{2}$ satisfy:

$$
\begin{aligned}
& \Omega_{1}\left(\xi_{2}, X\right)=2 t\left(d t \wedge \eta_{1}\right)\left(\xi_{2}, X\right)+t^{2} d \eta_{1}\left(\xi_{2}, X\right)=t^{2}\left(d \eta_{1}\right)\left(\xi_{2}, X\right) \text { for } X \text { tangent to } M ; \\
& \Omega_{1}\left(\xi_{2}, \partial t\right)=2 t\left(d t \wedge \eta_{1}\right)\left(\xi_{2}, \partial t\right)+t^{2} d \eta_{1}\left(\xi_{2}, \partial t\right)=-t \eta_{1}\left(\xi_{2}\right) ; \\
& \Omega_{2}\left(\xi_{1}, X\right)=2 t\left(d t \wedge \eta_{2}\right)\left(\xi_{1}, X\right)+t^{2} d \eta_{2}\left(\xi_{1}, X\right)=t^{2}\left(d \eta_{2}\right)\left(\xi_{1}, X\right) \text { for } X \text { tangent to } M ; \\
& \Omega_{2}\left(\xi_{1}, \partial t\right)=2 t\left(d t \wedge \eta_{2}\right)\left(\xi_{1}, \partial t\right)+t^{2} d \eta_{2}\left(\xi_{1}, \partial t\right)=-t \eta_{2}\left(\xi_{1}\right) .
\end{aligned}
$$

Then, by Theorems 4.2 and 4.1, we get

$\left(\eta_{1}, \eta_{2}\right)$ is a taut contact hyperbola (resp. circle) if and only if the corresponding symplectic 2 -forms $\left(\Omega_{1}, \Omega_{2}\right)$ satisfy $\Omega_{2}\left(\xi_{1}, \cdot\right)=\Omega_{1}\left(\xi_{2}, \cdot\right) \neq 0\left(\operatorname{resp} . \Omega_{2}\left(\xi_{1}, \cdot\right)=-\Omega_{1}\left(\xi_{2}, \cdot\right) \neq 0\right)$ in any point.

\section{Taut contact 2-hyperboloid}

Recall (cf. [13]) that a contact sphere on a three-manifold $M$ is a triple of contact 1-forms $\left(\eta_{1}, \eta_{2}, \eta_{3}\right)$ such that any linear combination $\left(a_{1} \eta_{1}+a_{2} \eta_{2}+a_{3} \eta_{3}\right), a \in \mathbb{S}^{2}$, is a contact form; moreover, it is taut if the volume forms $\eta_{a} \wedge\left(d \eta_{a}\right)$ on $M$ are equal for every $a \in \mathbb{S}^{2}$; moreover in this case the 1-forms $\left(\eta_{1}, \eta_{2}, \eta_{3}\right)$ parallelize the three-manifold $M$.

Now, consider the surface

$$
H_{r}^{2}: a_{1}^{2}-a_{2}^{2}-a_{3}^{2}=r, r \pm 1,
$$

that is, $H_{-1}^{2}$ is an one-sheeted hyperboloid and $H_{1}^{2}$ is a two-sheeted hyperboloid. In analogy with the definition of (taut) contact 2-sphere we give the following definition. We say that a triple of contact 1 -forms $\left(\eta_{1}, \eta_{2}, \eta_{3}\right)$ on a three-manifold $M$, is a contact 2-hyperboloid if the 1 -form

$$
\eta_{a}=a_{1} \eta_{1}+a_{2} \eta_{2}+a_{3} \eta_{3} \text { is a contact form for any } a \in H_{r}^{2} .
$$

This definition implies that any non-trivial linear combination $\eta_{a}=a_{1} \eta_{1}+a_{2} \eta_{2}+a_{3} \eta_{3}$ with constant coefficients $\left(a_{1}, a_{2}, a_{3}\right), a_{1}^{2}-a_{2}^{2}-a_{3}^{2} \neq 0$, is again a contact form. We call the 
triple $\left(\eta_{1}, \eta_{2}, \eta_{3}\right)$ a taut contact 2-hyperboloid if the volume forms $r\left(\eta_{a} \wedge d \eta_{a}\right)$ are equal for every $a \in H_{r}^{2}$, that is,

$$
\eta_{a} \wedge d \eta_{a}=r \eta_{1} \wedge d \eta_{1} \quad \text { for every } a \in H_{r}^{2}
$$

Besides, we note that

$$
\begin{aligned}
\eta_{a} \wedge d \eta_{a}= & a_{1}^{2} \eta_{1} \wedge d \eta_{1}+a_{2}^{2} \eta_{2} \wedge d \eta_{2}+a_{3}^{2} \eta_{3} \wedge d \eta_{3}+a_{1} a_{2}\left(\eta_{1} \wedge d \eta_{2}+\eta_{2} \wedge d \eta_{1}\right) \\
& +a_{1} a_{3}\left(\eta_{1} \wedge d \eta_{3}+\eta_{3} \wedge d \eta_{1}\right)+a_{2} a_{3}\left(\eta_{3} \wedge d \eta_{2}+\eta_{2} \wedge d \eta_{3}\right)
\end{aligned}
$$

Consequently, we get

Proposition 5.1 A triple of contact 1-forms $\left(\eta_{1}, \eta_{2}, \eta_{3}\right)$ on a three-manifold $M$ is a taut contact 2-hyperboloid if and only if $\left(\eta_{1}, \eta_{2}\right)$ and $\left(\eta_{1}, \eta_{3}\right)$ are taut contact hyperbola and the other pair $\left(\eta_{2}, \eta_{3}\right)$ is a taut contact circle.

Remark 5.2 We note that a triple of contact 1 -forms $\left(\eta_{1}, \eta_{2}, \eta_{3}\right)$ on a three-manifold $M$ defines a taut contact 2 -sphere if and only if $\left(\eta_{1}, \eta_{2}\right),\left(\eta_{1}, \eta_{3}\right)$ and $\left(\eta_{2}, \eta_{3}\right)$ are taut contact circles.

A difference, with respect to the taut contact 2-spheres, is that: in general the 1-forms $\eta_{1}, \eta_{2}, \eta_{3}$ that define a taut contact 2-hyperboloid are not linearly independent. In fact, we have the following.

Example 5.3 On the torus $\mathbb{T}^{3}$ the contact 1 -forms

$$
\eta_{1}=(\cos z) d x-(\sin z) d y, \quad \eta_{2}=(\cos z) d x+(\sin z) d y \text { and } \eta_{3}=(\sin z) d x-(\cos z) d y,
$$

are linearly dependent and define a taut contact 2-hyperboloid. More precisely: $\left(\eta_{1}, \eta_{2}\right)$, $\left(\eta_{1}, \eta_{3}\right)$ are taut contact hyperbola, and $\left(\eta_{2}, \eta_{3}\right)$ is a taut contact circle.

Now, we give the following

Proposition 5.4 Let $\left(\eta_{1}, \eta_{2}, \eta_{3}\right)$ be a coframe of contact 1-forms on a three-manifold M. Denote by $\left(e_{1}, e_{2}, e_{3}\right)$ the frame dual of $\left(\eta_{1}, \eta_{2}, \eta_{3}\right)$. Then, $\left(\eta_{1}, \eta_{2}, \eta_{3}\right)$ is a taut contact 2 -hyperboloid if and only if there exist three 1 -forms $\beta_{1}, \beta_{2}, \beta_{3}$ and a nonzero smooth function $\lambda$ such that

$$
\left\{\begin{array}{l}
d \eta_{1}=\beta_{1} \wedge \eta_{1}+\lambda \eta_{2} \wedge \eta_{3} \\
d \eta_{2}=\beta_{2} \wedge \eta_{2}-\lambda \eta_{3} \wedge \eta_{1} \\
d \eta_{3}=\beta_{3} \wedge \eta_{3}-\lambda \eta_{1} \wedge \eta_{2}
\end{array}\right.
$$

where the 1-forms $\beta_{i}$ satisfy

$$
\beta_{i}\left(e_{i}\right)=0 \text { and } \beta_{i}\left(e_{j}\right)=\beta_{k}\left(e_{j}\right) \text { for any }(i, j, k) \text { permutation of }(1,2,3) .
$$

In particular, $\beta_{1}=\beta_{2}=\beta_{3}$ if and only if $\beta_{i}=0$ for any $i=1,2$, 3. If this is the case, then the function $\lambda$ is a constant $\neq 0$. 
Proof Since $\left(\eta_{1}, \eta_{2}, \eta_{3}\right)$ is a coframe, we put

$$
\left\{\begin{array}{c}
d \eta_{1}=f_{1} \eta_{1} \wedge \eta_{2}+f_{2} \eta_{2} \wedge \eta_{3}+f_{3} \eta_{3} \wedge \eta_{1}, \\
d \eta_{2}=g_{1} \eta_{1} \wedge \eta_{2}+g_{2} \eta_{2} \wedge \eta_{3}+g_{3} \eta_{3} \wedge \eta_{1}, \\
d \eta_{3}=h_{1} \eta_{1} \wedge \eta_{2}+h_{2} \eta_{2} \wedge \eta_{3}+h_{3} \eta_{3} \wedge \eta_{1}
\end{array}\right.
$$

where $f_{i}, g_{i}, h_{i}$ are smooth functions. Then, the conditions

$$
\eta_{1} \wedge d \eta_{1}=-\eta_{2} \wedge d \eta_{2}=-\eta_{3} \wedge d \eta_{3}
$$

are equivalent to the conditions $f_{2}=-g_{3}=-h_{1}=\lambda$, where $\lambda$ is a nowhere zero function. Moreover, the conditions

$$
0=\eta_{1} \wedge d \eta_{2}+\eta_{2} \wedge d \eta_{1}=\eta_{1} \wedge d \eta_{3}+\eta_{3} \wedge d \eta_{1}=\eta_{2} \wedge d \eta_{3}+\eta_{3} \wedge d \eta_{2}
$$

are equivalent to the conditions $g_{2}=-f_{3}=-\lambda_{3}, h_{2}=-f_{1}=\lambda_{2}, h_{3}=-g_{1}=-\lambda_{1}$. Thus, $\left(\eta_{1}, \eta_{2}, \eta_{3}\right)$ is a taut contact 2-hyperboloid if and only if

$$
\left\{\begin{array}{l}
d \eta_{1}=\beta_{1} \wedge \eta_{1}+\lambda \eta_{2} \wedge \eta_{3} \\
d \eta_{2}=\beta_{2} \wedge \eta_{2}-\lambda \eta_{3} \wedge \eta_{1} \\
d \eta_{3}=\beta_{3} \wedge \eta_{3}-\lambda \eta_{1} \wedge \eta_{2}
\end{array}\right.
$$

where

$$
\beta_{1}=\left(\lambda_{2} \eta_{2}+\lambda_{3} \eta_{3}\right), \quad \beta_{2}=\left(\lambda_{1} \eta_{1}+\lambda_{3} \eta_{3}\right) \text { and } \beta_{3}=\left(\lambda_{1} \eta_{1}+\lambda_{2} \eta_{2}\right)
$$

Consequently, the 1 -forms $\beta_{i}$ satisfy $(*)$; moreover, $\beta_{1}=\beta_{2}=\beta_{3}$ if and only if $\beta_{1}=\beta_{2}=\beta_{3}=0$. In this case, we have

$$
0=d^{2} \eta_{1}=d \lambda \wedge \eta_{2} \wedge \eta_{3}+\lambda\left(d \eta_{2}\right) \wedge \eta_{3}-\lambda \eta_{2} \wedge d \eta_{3}=d \lambda \wedge \eta_{2} \wedge \eta_{3} \Rightarrow e_{1}(\lambda)=0
$$

Analogously, $e_{2}(\lambda)=e_{3}(\lambda)=0$, and so $\lambda$ is a constant.

Corollary 5.5 A simply connected three-manifold $M$ admits a taut contact 2-hyperboloid $\left(\eta_{1}, \eta_{2}, \eta_{3}\right)$, with the corresponding Reeb vector fields $\left(\xi_{1}, \xi_{2}, \xi_{3}\right)$ that constitute the frame dual of the coframe $\left(\eta_{1}, \eta_{2}, \eta_{3}\right)$, if and only if $M$ is the Lie group $\widetilde{S L}(2, \mathbb{R})$.

Proof In the Proposition 5.4, the condition $\beta_{1}=\beta_{2}=\beta_{3}$, i.e., $\beta_{i}=0$ for any $i=1,2,3$, is equivalent to the condition that the vector fields $e_{i}$ are the Reeb vector fields of the contact forms $\eta_{i}, i=1,2,3$. Then, if $\left(\eta_{1}, \eta_{2}, \eta_{3}\right)$ is a taut contact 2 -hyperboloid with the corresponding Reeb vector fields $\left(\xi_{1}, \xi_{2}, \xi_{3}\right)$ that constitute the frame dual of the coframe $\left(\eta_{1}, \eta_{2}, \eta_{3}\right)$, from (5.1) we get that the 1 -forms $\beta_{i}$ vanish and so the Reeb vector fields satisfy

$$
\left[\xi_{1}, \xi_{2}\right]=\lambda \xi_{3}, \quad\left[\xi_{2}, \xi_{3}\right]=-\lambda \xi_{1}, \quad\left[\xi_{3}, \xi_{1}\right]=\lambda \xi_{2}, \lambda=\text { const. } \neq 0 .
$$

Therefore, $M$ admits a Lie group structure isomorphic to $\widetilde{S L}(2, \mathbb{R})$.

Conversely, if we consider the Lie group $S L(2, \mathbb{R})$, by using the notations of the Example 3.9 , we have the 1 -forms $\left(\eta_{1}, \eta_{2}\right)$ and $\left(\eta_{1}, \eta_{3}\right)$ are left invariant contact hyperbolas and $\left(\eta_{2}, \eta_{3}\right)$ is a left invariant taut contact circle. Then, by Proposition 5.1, we get that the 1-forms $\left(\eta_{1}, \eta_{2}, \eta_{3}\right)$ define a left invariant taut contact 2-hyperboloid. Moreover, 
the corresponding Reeb vector fields $\left(\xi_{1}, \xi_{2}, \xi_{3}\right)$ constitute the frame dual of the coframe $\left(\eta_{1}, \eta_{2}, \eta_{3}\right)$.

Remark 5.6 In the compact case, taut contact 2-spheres exist only on left quotients of the three-sphere group $\mathbb{S}^{3}=S U(2)$ ( [13], Theorem 1.10). In particular, the torus $\mathbb{t}^{3}$ does not admit a taut contact 2 -sphere, however it admits a taut contact 2-hyperboloid.

\section{Taut contact hyperbolas and the Chern-Hamilton energy functional}

Let $(M, \eta)$ be an oriented compact contact manifold. Denote by $\mathcal{M}(\eta)$ the set of all Riemannian metrics associated to the contact form $\eta$ and by $\mathcal{A}(\eta)$ the set of all almost CR structures $J$ for which the Levi form is positive definite. The sets $\mathcal{M}(\eta)$ and $\mathcal{A}(\eta)$ can be identified (cf., for example, Proposition 8 of [25]). Tanno [26] considered the Dirichlet energy

$$
E(g)=\int_{M}\|\tau\|^{2} d v, \quad \tau=\mathcal{L}_{\xi} g,
$$

defined for any $g \in \mathcal{M}(\eta)$. Then, he found the critical point condition ( [26], Theorem 5.1)

$$
\nabla_{\xi} \tau=2 \tau \varphi, \text { equivalently } \nabla_{\xi} h=-2 \varphi h .
$$

The Dirichlet energy (6.1) was first studied by Chern and Hamilton [9] for compact contact three-manifolds as a functional defined on the set $\mathcal{A}(\eta)$ (there was an error in their calculation of the critical point condition, as was pointed out by Tanno). This functional is known in literature also with the name of Chern-Hamilton energy functional. Moreover, since $\operatorname{Ric}(\xi, \xi)=2 n-t r h^{2}=2 n-\|\tau\|^{2} / 4$, the functional (6.1) is equivalent to the functional $L(g)=\int_{M} \operatorname{Ric}(\xi, \xi) d v$ studied in general dimension, for compact regular contact manifold, by Blair ( [3], Section 10.3). We note that $K$-contact metrics and Sasakian metrics are trivial critical metrics, besides we note that the critical point condition (6.2) has a tensorial character, so it holds also in the non-compact case. On the other hand, the sphere $\mathbb{S}^{3}$ admits a Sasakian structure, therefore: in general a Sasakian three-manifold fails to admit a taut contact hyperbola.

Next, we show that the critical point condition (6.2) is a sufficient condition for the existence of a taut contact hyperbola on a non-Sasakian contact metric three-manifold. In fact, we have the following.

Theorem 6.1 Let $(M, \eta, g, \varphi, \xi)$ be a non-Sasakian contact metric three-manifold, that is, the torsion $\tau \neq 0$ at any point. If the metric $g$ satisfies the critical point condition for the Dirichlet energy functional (6.1), then $M$ admits a taut contact hyperbola.

Proof Let $(M, \eta, g, \varphi, \xi)$ be a non-Sasakian contact metric three-manifold. Let $\left\{e_{1}, e_{2}=\varphi e_{1}, \xi\right\}$ be an orthonormal basis of smooth eigenvectors for $h$ with $h \xi=0$, $h e_{1}=\lambda e_{1}, h e_{2}=-\lambda e_{2}, \lambda$ being the positive eigenvalue. Since the three eigenvalues $0, \lambda,-\lambda$ of $h$ are everywhere distinct, the corresponding line fields are global and by the orientability the basis can be taken to be global. Let $\eta_{1}, \eta_{2}$ be the 1 -forms $g$-dual to $e_{1}$ and $e_{2}$, respectively, and hence $\left(\eta_{1}, \eta_{2}, \eta\right)$ is a global basis of 1 -forms. Using (2.1), we have 


$$
\nabla_{e_{1}} \xi=-\varphi e_{1}-\varphi h e_{1}=-(1+\lambda) e_{2} \quad \text { and } \quad \nabla_{e_{2}} \xi=(1-\lambda) e_{1} .
$$

By straightforward computation and using $\nabla_{\xi} \xi=0$, we get

$$
\nabla_{\xi} e_{1}=a e_{2} \quad \text { and } \quad \nabla_{\xi} e_{2}=-a e_{1},
$$

where $a=g\left(\nabla_{\xi} e_{1}, e_{2}\right)$ is a smooth function. Moreover, $\left(\nabla_{\xi} h\right) \xi=0$ and by using (6.4) we obtain

$$
\left(\nabla_{\xi} h\right) e_{1}=\nabla_{\xi} h e_{1}-h\left(\nabla_{\xi} e_{1}\right)=\xi(\lambda) e_{1}+2 a \lambda e_{2}
$$

and

$$
\left(\nabla_{\xi} h\right) e_{2}=\left(\nabla_{\xi} h\right) \varphi e_{1}=-\varphi\left(\nabla_{\xi} h\right) e_{1}=-\xi(\lambda) e_{2}+2 a \lambda e_{1}
$$

Thus,

$$
\left(\nabla_{\xi} h\right)=-2 a h \varphi+(\xi(\lambda) / \lambda) h .
$$

Consequently, since $g$ satisfies the critical point condition (6.2), we have $a=-1$ and $\xi(\lambda)=0$. Thus, (6.4) becomes

$$
\nabla_{\xi} e_{1}=-e_{2} \quad \text { and } \quad \nabla_{\xi} e_{2}=e_{1} .
$$

Then, by using (6.3) and (6.5) we have

$$
\begin{aligned}
& \eta_{1} \wedge d \eta_{1}\left(\xi, e_{1}, e_{2}\right)=-\left(d \eta_{1}\right)\left(\xi, e_{2}\right)=\frac{1}{2} g\left(e_{1}, \nabla_{\xi} e_{2}-\nabla_{e_{2}} \xi\right)=\frac{\lambda}{2}>0, \\
& \eta_{2} \wedge d \eta_{2}\left(\xi, e_{1}, e_{2}\right)=\left(d \eta_{2}\right)\left(\xi, e_{1}\right)=-\frac{1}{2} g\left(e_{2}, \nabla_{\xi} e_{1}-\nabla_{e_{1}} \xi\right)=-\frac{\lambda}{2}<0, \\
& \eta_{1} \wedge d \eta_{2}\left(\xi, e_{1}, e_{2}\right)=-\left(d \eta_{2}\right)\left(\xi, e_{2}\right)=\frac{1}{2} g\left(e_{2}, \nabla_{\xi} e_{2}-\nabla_{e_{2}} \xi\right)=0, \\
& \eta_{2} \wedge d \eta_{1}\left(\xi, e_{1}, e_{2}\right)=\left(d \eta_{1}\right)\left(\xi, e_{1}\right)=-\frac{1}{2} g\left(e_{1}, \nabla_{\xi} e_{1}-\nabla_{e_{1}} \xi\right)=0,
\end{aligned}
$$

Therefore, by using Proposition 3.3, the 1 -forms $\left(\eta_{1}, \eta_{2}\right)$ define a taut contact hyperbola.

Following Y. Eliashberg [11], a contact manifold $(M, \eta)$ is called overtwisted if there exists an embedded disk $D$ in $M$ such that $T_{p} D=\operatorname{ker} \eta_{p}$ for all $p \in \partial D$. It is called tight if it is not overtwisted. Moreover, the contact distribution is called universally tight if even its lift to the universal cover of $M$ is tight. Recently, S. Hozoori ( [18], Theorem 1.4) proved, in the compact case, that a conformally Anosov contact three-manifold is universally tight. On the other hand, by proof of Theorem 6.1 we note that the intersection of the bi-contact structure $\left(\eta_{1}, \eta_{2}\right)$ is given by $\mathbb{R} \xi$ and thus, by [21], $(M, \operatorname{ker} \eta)$ is a conformally Anosov contact three-manifold. Therefore, we have the following

Corollary 6.2 Let $(M, \eta, g, \varphi, \xi)$ be a compact non-Sasakian contact metric three-manifold. If the metric $g$ is a critical metric for the Dirichlet energy functional, then $(M, \operatorname{ker} \eta)$ is universally tight. 
Next, we exhibit an example of compact non-Sasakian contact metric three-manifold with the contact Riemannian metric $g$ critical for the Dirichlet energy (6.1).

Example 6.3 Let $(M, g)$ be a compact 2-dimensional Riemannian manifold of constant sectional curvature $k<0$. By Theorem 7 and Corollary 3 of the paper [1], we get that the unit tangent sphere bundle $T_{1} M$ admits a family of non-Sasakian contact metric structures $\left(\tilde{\eta}_{a}, \tilde{G}_{a}\right)$, depending on one parameter $a>0$, satisfying the critical point condition (6.2), where the critical metric $\tilde{G}_{a}$ is a Riemannian $g$-natural metric. In particular, for $a=1 / 4$ and $k=-1,\left(\tilde{\eta}_{a}, \tilde{G}_{a}\right)$ is the standard (non-Sasakian) contact Riemannian structure on $T_{1} M$ satisfying the critical point condition (6.2) ( [3], Th. 10.13, p.208), where $\tilde{G}_{a}$ is the classic Sasaki metric $\tilde{G}_{S}$. In general, for $a>0, \tilde{G}_{a}$ is a metric of Kaluza-Klein type, i.e., horizontal and tangential lifts are mutually orthogonal with respect to $\tilde{G}_{a}$. To note that the Sasaki metric on $T_{1} M$, in general, is not Sasakian in the sense of the contact Riemannian geometry.

\section{Geometry of bi-contact metric structures}

In this section, we study the geometry of a three-manifold determined by the existence of a bi-contact metric structure. The following theorem, which can be considered as a more complete presentation of Theorem 3.6 of [24], will be very useful for this study.

Theorem 7.1 Let $\left(\eta_{1}, \eta_{2}\right)$ be a pair of contact forms on a three-manifold $M$, with Reeb vector fields $\left(\xi_{1}, \xi_{2}\right), \xi_{2} \neq \pm \xi_{1}$. Then, the following are equivalent.

(I) $\quad\left(\eta_{1}, \eta_{2}\right)$ defines a bi-contact metric structure, i.e., there exists a Riemannian metric $g$ for which $\left(\eta_{1}, \eta_{2}, g\right)$ is a bi-contact metric structure.

(II) $\quad\left(\eta_{1}, \eta_{2}\right)$ is a $(-\varepsilon)$-Cartan structure, i.e.,

$$
\eta_{1} \wedge d \eta_{2}=\eta_{2} \wedge d \eta_{1}=0, \quad \eta_{2} \wedge d \eta_{2}=-\varepsilon \eta_{1} \wedge d \eta_{1}, \varepsilon= \pm 1
$$

(III) There exists a unique 1-form $\eta_{3}$ such that

$$
d \eta_{1}=-2 \eta_{2} \wedge \eta_{3}, \quad d \eta_{2}=-2 \varepsilon \eta_{1} \wedge \eta_{3}, \quad d \eta_{3}=2 \kappa \eta_{2} \wedge \eta_{1},
$$

where the smooth function $\kappa=\left(d \eta_{3}\right)\left(\xi_{2}, \xi_{1}\right)$ satisfies $d \kappa \wedge \eta_{1} \wedge \eta_{2}=0$.

Proof $(I) \Rightarrow(I I)$.

Let $\left(\eta_{1}, \eta_{2}, g\right)$ be a bi-contact metric structure and $\left(\eta_{1}, \xi_{1}, \varphi_{1}, g\right),\left(\eta_{2}, \xi_{2}, \varphi_{2}, g\right)$ the corresponding contact metric structure with $g\left(\xi_{1}, \xi_{2}\right)=0$. Consider the vector field $\xi_{3}=\varphi_{1} \xi_{2}=\varepsilon \varphi_{2} \xi_{1}, \varepsilon= \pm 1$ (cf. Definition 2.1). Then $\left(\xi_{1}, \xi_{2}, \xi_{3}\right)$ is a global orthonormal basis and $\eta_{i}\left(\xi_{j}\right)=\delta_{i j}$ for $i=1,2$ and $j=1,2,3$. Consequently, $\eta_{1} \wedge d \eta_{2}=0=\eta_{2} \wedge d \eta_{1}$ and

$$
\left(\eta_{2} \wedge d \eta_{2}\right)\left(\xi_{1}, \xi_{2}, \xi_{3}\right)=-\eta_{2}\left(\xi_{2}\right) d \eta_{2}\left(\xi_{1}, \xi_{3}\right)=-g\left(\xi_{1}, \varphi_{2} \xi_{3}\right)=\varepsilon,\left(\eta_{1} \wedge d \eta_{1}\right)\left(\xi_{1}, \xi_{2}, \xi_{3}\right)=\ldots=1
$$

that is, $\eta_{2} \wedge d \eta_{2}=-\varepsilon \eta_{1} \wedge d \eta_{1}$.

$(I I) \Rightarrow(I I I)$.

By using (II), (3.1) and (3.3), we get that $\left(\eta_{1}, \eta_{2}\right)$ ia taut contact hyperbola (resp. circle) if $\varepsilon=1$ (resp. $\varepsilon=-1$ ). From Theorem 4.2 (if $\varepsilon=1$ ) and Theorem 4.1 (if $\varepsilon=-1$ ), we get that $\xi_{1}, \xi_{2}$ are linearly independent, $\eta_{1}\left(\xi_{2}\right)=\varepsilon \eta_{2}\left(\xi_{1}\right)$ and $d \eta_{1}\left(\xi_{2}, \cdot\right)=\varepsilon d \eta_{2}\left(\xi_{1}, \cdot\right) \neq 0$ 
everywhere. Then, $\eta_{1} \wedge d \eta_{2}=0=\eta_{2} \wedge d \eta_{1}$ implies $\eta_{1}\left(\xi_{2}\right)=\eta_{2}\left(\xi_{1}\right)=0$. Now, consider the 1 -form

$$
\eta_{3}=d \eta_{1}\left(\cdot, \xi_{2}\right)=\varepsilon d \eta_{2}\left(\cdot, \xi_{1}\right) \neq 0 \text { everywhere. }
$$

Then, $\eta_{3}\left(\xi_{2}\right)=\eta_{3}\left(\xi_{1}\right)=0$ and there exists a vector field $\xi_{3}$ such that $\eta_{3}\left(\xi_{3}\right)=1,\left(\xi_{1}, \xi_{2}, \xi_{3}\right)$ is a basis and thus $\eta_{1} \wedge \eta_{2} \wedge \eta_{3}$ is a volume form and $\left(\eta_{1} \wedge \eta_{2}, \eta_{1} \wedge \eta_{3}, \eta_{2} \wedge \eta_{3}\right)$ is a basis of 2-forms. Since

$$
d \eta_{1}\left(\xi_{1}, \cdot\right)=d \eta_{2}\left(\xi_{2}, \cdot\right)=0, d \eta_{1}\left(\xi_{2}, \xi_{3}\right)=-\eta_{3}\left(\xi_{3}\right)=-1 \text { and } d \eta_{2}\left(\xi_{1}, \xi_{3}\right)=-\varepsilon \eta_{3}\left(\xi_{3}\right)=-\varepsilon,
$$

we get

$$
d \eta_{1}=-2 \eta_{2} \wedge \eta_{3}, \quad d \eta_{2}=-2 \varepsilon \eta_{1} \wedge \eta_{3}
$$

Finally, by using (7.1), we have

$$
0=d \eta_{2} \wedge \eta_{3}=\eta_{2} \wedge d \eta_{3} \quad \text { and } \quad 0=d \eta_{1} \wedge \eta_{3}=\eta_{1} \wedge d \eta_{3}
$$

and hence $d \eta_{3}=2 \kappa \eta_{2} \wedge \eta_{3}$ where $\kappa$ is a smooth function satisfying $d \kappa \wedge \eta_{1} \wedge \eta_{2}=0$, that is, $\kappa_{3}=0$ where we put $d \kappa=\sum_{i} \kappa_{i} \eta_{i}$. The 1 -form $\eta_{3}$ satisfying (III) is unique because from (7.1) one gets that $\eta_{3}=-d \eta_{1}\left(\xi_{2}, \cdot\right)=-\varepsilon d \eta_{2}\left(\xi_{1}, \cdot\right)$.

\section{$(I I I) \Rightarrow(I)$.}

Let $\left(\xi_{1}, \xi_{2}, \xi_{3}\right)$ be the triple of vector fields dual to the basis $\left(\eta_{1}, \eta_{2}, \eta_{3}\right)$ of 1-forms. We note that $\xi_{1}, \xi_{2}$ are necessarily the Reeb vector fields of $\eta_{1}, \eta_{2}$, respectively. Moreover by the usual formulae, the dual of the equations of (III) are

$$
\left[\xi_{1}, \xi_{2}\right]=2 \kappa \xi_{3}, \quad\left[\xi_{2}, \xi_{3}\right]=2 \xi_{1}, \quad\left[\xi_{3}, \xi_{1}\right]=-2 \varepsilon \xi_{2},
$$

where $\kappa$ is a smooth function. Now, we consider the Riemannian metric $g$ defined by $g\left(\xi_{i}, \xi_{j}\right)=\delta_{i j}$. Then $\eta_{1}=g\left(\xi_{1}, \cdot\right)$ and $\eta_{2}=g\left(\xi_{2}, \cdot\right)$. Moreover, if we define the $(1,1)$-tensors $\varphi_{1}$ and $\varphi_{2}$ by

$$
\varphi_{1} \xi_{1}=0, \quad \varphi_{1} \xi_{2}=\xi_{3}, \quad \varphi_{1} \xi_{3}=-\xi_{2}, \quad \varphi_{2} \xi_{2}=0, \quad \varphi_{2} \xi_{1}=\varepsilon \xi_{3}, \quad \varphi_{2} \xi_{3}=-\varepsilon \xi_{1},
$$

then $\left(\eta_{1}, \xi_{1}, \varphi_{1}\right)$ and $\left(\eta_{2}, \xi_{2}, \varphi_{2}\right)$ are almost contact structures. Moreover, by using (7.2), we get $d \eta_{1}=g\left(\cdot, \varphi_{1}\right)$ and $d \eta_{2}=g\left(\cdot, \varphi_{2}\right)$, that is,$\left(\eta_{1}, \eta_{2}, g\right)$ is a bi-contact metric structure on $M$.

From Theorem 7.1 follows that for a bi-contact metric structure $\left(\eta_{1}, \eta_{2}, g\right)$ are uniquely determined the 1 -form $\eta_{3}=d \eta_{1}\left(\cdot, \xi_{2}\right)$ and the smooth function $\kappa=\left(d \eta_{3}\right)\left(\xi_{2}, \xi_{1}\right)$. In particular, we deduce

Corollary 7.2 If a $3 D$ Lie group $G$ admits a left invariant bi-contact metric structure, then $G$ is unimodular.

The next example shows that there exist taut contact hyperbolas/circles which do not define $(-\varepsilon)$-Cartan structures.

Example 7.3 Consider on $\mathbb{R}^{3}$ the 1 -forms

$$
\eta_{1}=(a y+b z) f(x) d x+d y \quad \text { and } \quad \eta_{2}=(\varepsilon b y+a z) f(x) d x+d z
$$


where $f(x)$ is a positive smooth function and $a, b \in \mathbb{R}, a, b \neq 0$. Then

$$
d \eta_{1}=a f(x) d y \wedge d x+b f(x) d z \wedge d x \quad \text { and } \quad d \eta_{2}=\varepsilon b f(x) d y \wedge d x+a f(x) d z \wedge d x .
$$

Consequently,

$$
\eta_{1} \wedge d \eta_{1}=b f(x) d x \wedge d y \wedge d z, \quad \eta_{2} \wedge d \eta_{2}=-\varepsilon \eta_{1} \wedge d \eta_{1} \neq 0
$$

and

$$
\eta_{1} \wedge d \eta_{2}=-\eta_{2} \wedge d \eta_{1}=a f(x) d x \wedge d y \wedge d z \neq 0 .
$$

So for $\varepsilon=1,\left(\eta_{1}, \eta_{2}\right)$ defines a taut contact hyperbola, and $\varepsilon=-1,\left(\eta_{1}, \eta_{2}\right)$ defines a taut contact circle. In both the cases $\left(\eta_{1}, \eta_{2}\right)$ does not define a $(-\varepsilon)$-Cartan structure. Besides, by Remark 4.5 , we get that on the four manifold $\mathbb{R}^{3} \times \mathbb{R}_{+}$the corresponding symplectic 2-forms define a symplectic pair for $\varepsilon=1$ and a conformal symplectic couple for $\varepsilon=-1$.

Remark 7.4 An interpretation of the function $\kappa$ in terms of the Webster scalar curvature. Consider the Webster scalar curvature $\mathcal{W}$ as defined by Chern and Hamilton [9] in their study on contact Riemannian three-manifolds. If $(M, \eta, g)$ is a contact Riemannian three-manifold, the Webster scalar curvature is given by ( [9], p.284)

$$
\mathcal{W}=(1 / 8)(w-\operatorname{Ric}(\xi, \xi)+4),
$$

where $w$ is the usual scalar curvature and $\operatorname{Ric}(\xi, \xi)$ is the Ricci curvature in the direction of the Reeb vector field $\xi$. We note that the generalized Tanaka-Webster scalar curvature $\hat{w}$ (cf. [26]) is eight times the Webster scalar curvature $\mathcal{W}$ as defined by Chern and Hamilton. Moreover, a compact simply connected regular Sasakian $(2 n+1)$-manifold is a principal $\mathbb{S}^{1}$ -bundle over a compact Kaehler manifold $B$ of complex dimension $n$, and the generalized Tanaka-Webster scalar curvature $\hat{w}$ is the scalar curvature of the Kaehler manifold $B$ ( [25] p.26). Of course, in dimension three, $B$ is a Riemann surface and hence the Webster scalar curvature $\mathcal{W}$ determines the Gaussian curvature $(4 \mathcal{W})$ and the Eulero-Poincaré characteristic of $B$.

Now, let $\left(\eta_{1}, \eta_{2}, g\right)$ be a bi-contact metric structure. Then, we have the following (cf. [24], p.224):

- if $\left(\eta_{1}, \eta_{2}\right)$ is a taut contact circle, i.e., $\varepsilon=-1$, the Webster scalar curvatures of $\left(\eta_{1}, g\right)$ and $\left(\eta_{2}, g\right)$ are given by the same function

$$
\mathcal{W}=(\kappa+1) / 2
$$

- if $\left(\eta_{1}, \eta_{2}\right)$ is a taut contact hyperbola, i.e., $\varepsilon=+1$, the Webster scalar curvatures of $\left(\eta_{1}, g\right)$ and $\left(\eta_{2}, g\right)$ are given, respectively, by the functions

$$
\mathcal{W}_{1}=(\kappa-1) / 2 \quad \text { and } \quad \mathcal{W}_{2}=-(\kappa+1) / 2
$$

So, in both the cases the function $\kappa$ determines the Webster curvature and it does not depend on the associated metric $g$. Therefore, we call $\kappa$ the Webster function of the $(-\varepsilon)$ -Cartan structure $\left(\eta_{1}, \eta_{2}\right)$. If $\left(\eta_{1}, \eta_{2}\right)$ is a taut contact circle, i.e., a Cartan structure, the Webster function $\kappa$ is invariant for an Euclidean rotation of constant angle (cf. [24]).

Now, we suppose that $\left(\eta_{1}, \eta_{2}\right)$ is a taut contact hyperbola and consider a hyperbolic rotation of constant angle of $\left(\eta_{1}, \eta_{2}\right)$, i.e., 


$$
\left(\eta_{1}^{\prime}=a_{1} \eta_{1}+a_{2} \eta_{2}, \eta_{2}^{\prime}=r\left(a_{2} \eta_{1}+a_{1} \eta_{2}\right), r= \pm 1, \text { with } a=\left(a_{1}, a_{2}\right) \in \mathbb{M}_{1}^{1}\right.
$$

Since

$$
\begin{aligned}
& \eta_{1}^{\prime} \wedge d \eta_{1}^{\prime}=\left(a_{1}^{2}-a_{2}^{2}\right) \eta_{1} \wedge d \eta_{1}=\eta_{1} \wedge d \eta_{1}, \quad \eta_{2}^{\prime} \wedge d \eta_{2}^{\prime}=\left(a_{2}^{2}-a_{1}^{2}\right) \eta_{1} \wedge d \eta_{1}=-\eta_{1} \wedge d \eta_{1} \\
& \eta_{1}^{\prime} \wedge d \eta_{2}^{\prime}=r\left(a_{1}^{2}-a_{2}^{2}\right) \eta_{1} \wedge d \eta_{2}=0, \quad \eta_{2}^{\prime} \wedge d \eta_{1}^{\prime}=r\left(a_{2}^{2}-a_{1}^{2}\right) \eta_{1} \wedge d \eta_{2}=0
\end{aligned}
$$

then $\left(\eta_{1}^{\prime}, \eta_{2}^{\prime}\right)$ is again a $(-1)$-Cartan structure. Moreover, the corresponding Reeb vector fields of the contact forms $\left(\eta_{1}^{\prime}, \eta_{2}^{\prime}\right)$ are $\xi_{1}^{\prime}=a_{1} \xi_{1}-a_{2} \xi_{2}$ and $\xi_{2}^{\prime}=-r\left(a_{2} \xi_{1}-a_{1} \xi_{2}\right)$. Consequently,

$$
\eta_{3}^{\prime}=-d \eta_{1}^{\prime}\left(\xi_{2}^{\prime}, \cdot\right)=r\left(a_{1} d \eta_{1}+a_{2} d \eta_{2}\right)\left(a_{2} \xi_{1}-a_{1} \xi_{2}, \cdot\right)=r \eta_{3}=\ldots=-d \eta_{2}^{\prime}\left(\xi_{1}^{\prime}, \cdot\right),
$$

and thus $d \eta_{3}^{\prime}=r d \eta_{3}=2 r \kappa \eta_{2} \wedge \eta_{1}=\ldots=2 \kappa \eta_{2}^{\prime} \wedge \eta_{1}^{\prime}$. Therefore, the Webster function of the $(-1)$-Cartan structure $\left(\eta_{1}^{\prime}, \eta_{2}^{\prime}\right)$ is $\kappa^{\prime}=\kappa$, i.e., for a $(-1)$-Cartan structure, the Webster function $\kappa$ is invariant for a hyperbolic rotation of constant angle.

About the 1-form $\eta_{3}$ and the Webster function $\kappa$, we have the following

Theorem 7.5 Let $\left(\eta_{1}, \eta_{2}, g\right)$ be a bi-contact metric structure on the three-manifold $M$. Then, for the 1-form $\eta_{3}$ hold the following properties.

(1) $\eta_{3}$ is a Killing 1-form (with respect to $g$ ) if and only if $\left(\eta_{1}, \eta_{2}\right)$ is a taut contact circle.

(2) $\eta_{3}$ is a contact form if and only if the Webster function $\kappa \neq 0$ everywhere.

(3) If $\eta_{3}$ is a contact form, then

$$
\left(\eta=-\bar{\varepsilon} \eta_{3}, g_{\kappa}=\varepsilon \bar{\varepsilon} \kappa g+(1-\varepsilon \bar{\varepsilon} \kappa) \eta \otimes \eta\right), \text { where } \bar{\varepsilon}=\varepsilon(\operatorname{sign\kappa }),
$$

is a contact metric structure of the three-manifold $M$, and it is a Sasakian structure if and only if $\left(\eta_{1}, \eta_{2}\right)$ is a taut contact circle.

Proof Let $\left(\eta_{1}, \eta_{2}, g\right)$ be a bi-contact metric structure on the three-manifold $M$. Denote by $\left(\eta_{i}, \varphi_{i}, \xi_{i}, g\right), i=1,2$, the corresponding contact metric structures with $g\left(\xi_{1}, \xi_{2}\right)=0$. The 1 -form $\eta_{3}$ defined by (III) of Theorem 7.1 is given by $\eta_{3}=-d \eta_{1}\left(\xi_{2}, \cdot\right) \neq 0$ everywhere, and thus

$$
\eta_{3}=d \eta_{1}\left(\cdot, \xi_{2}\right)=g\left(\cdot, \varphi_{1} \xi_{2}\right)=g\left(\xi_{3}, \cdot\right), \quad \text { where } \xi_{3}:=\varphi_{1} \xi_{2}=\varepsilon \varphi_{2} \xi_{1} .
$$

Moreover, $\quad g\left(\xi_{3}, \xi_{3}\right)=\eta_{3}\left(\xi_{3}\right)=g\left(\varphi_{1} \xi_{2}, \varphi_{1} \xi_{2}\right)=1, \quad g\left(\xi_{1}, \xi_{3}\right)=-g\left(\varphi_{1} \xi_{1}, \xi_{2}\right)=0 \quad$ and $g\left(\xi_{2}, \xi_{3}\right)=-\varepsilon g\left(\varphi_{2} \xi_{2}, \xi_{3}\right)=0$. So $\left(\xi_{1}, \xi_{2}, \xi_{3}\right)$ is an orthonormal basis, dual to the basis of 1 -forms $\left(\eta_{1}, \eta_{2}, \eta_{3}\right)$. Moreover, by proof of Theorem 7.1, the basis $\left(\xi_{1}, \xi_{2}, \xi_{3}\right)$ satisfies (7.2). Consequently, the fundamental tensors $h_{1}=(1 / 2) \mathcal{L}_{\xi_{1}} \varphi_{1}$ and $h_{2}=(1 / 2) \mathcal{L}_{\xi_{2}} \varphi_{2}$ of the contact metric structures $\left(\eta_{1}, g\right),\left(\eta_{2}, g\right)$, respectively, satisfy

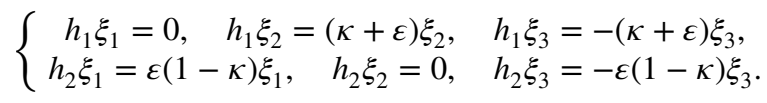

Now, recall that a 1-form $\eta$ on a Riemannian manifold $(M, g)$ is a Killing form if and only if $i(X) d \eta=\nabla_{X} \eta$ for any vector field $X$ on $M$, 
where $\nabla$ is the Levi-Civita connection of the metric $g$. Then, in our case, by using the notations introduced before, $\eta_{3}$ is a Killing form if and only if

$$
d \eta_{3}\left(\xi_{i}, \xi_{j}\right)=\left(\nabla_{\xi_{i}} \eta_{3}\right) \xi_{j} \text { for } i, j=1,2,3 .
$$

By (III) of Theorem 7.1, $d \eta_{3}=2 \kappa \eta_{2} \wedge \eta_{1}$, thus

$$
d \eta_{3}\left(\xi_{i}, \xi_{j}\right)=0 \text { when } i=3 \operatorname{orj}=3 \text {, and } d \eta_{3}\left(\xi_{1}, \xi_{2}\right)=-\kappa \text {. }
$$

Moreover, we have

$$
\left(\nabla_{\xi_{i}} \eta_{3}\right) \xi_{j}=-g\left(\xi_{3}, \nabla_{\xi_{i}} \xi_{j}\right)
$$

Since, by using (2.1), (7.4), and (7.2), we get

$$
\left\{\begin{array}{l}
\nabla_{\xi_{1}} \xi_{1}=0, \quad \nabla_{\xi_{2}} \xi_{1}=-(1+\kappa+\varepsilon) \xi_{3}, \quad \nabla_{\xi_{3}} \xi_{1}=(1-\kappa-\varepsilon) \xi_{2}, \\
\nabla_{\xi_{2}} \xi_{2}=0, \quad \nabla_{\xi_{1}} \xi_{2}=-(1-\kappa+\varepsilon) \xi_{3}, \quad \nabla_{\xi_{3}} \xi_{2}=(\varepsilon-1+\kappa) \xi_{1}, \\
\nabla_{\xi_{1}} \xi_{3}=(1-\kappa+\varepsilon) \xi_{2}, \quad \nabla_{\xi_{2}} \xi_{3}=(1+\kappa+\varepsilon) \xi_{1}, \quad \nabla_{\xi_{3}} \xi_{3}=0 .
\end{array}\right.
$$

Then, we obtain

$\left(\nabla_{\xi_{1}} \eta_{3}\right) \xi_{2}=(1-\kappa+\varepsilon),\left(\nabla_{\xi_{2}} \eta_{3}\right) \xi_{1}=(1+\kappa+\varepsilon)$ and $\left(\nabla_{\xi_{i}} \eta_{3}\right) \xi_{j}=0$ in the other cases.

Therefore,

$$
d \eta_{3}\left(\xi_{i}, \xi_{j}\right)=\left(\nabla_{\xi_{i}} \eta_{3}\right) \xi_{j} \text { for } i, j=1,2,3 \Longleftrightarrow \varepsilon+1=0,
$$

that is, the property (1). Since $\eta_{3} \wedge d \eta_{3}=2 \kappa \eta_{3} \wedge \eta_{2} \wedge \eta_{1}$, we get the property (2).

Now, suppose that $\eta_{3}$ is a contact form, that is, the Webster function $\kappa \neq 0$ everywhere. Then, $\xi_{3}=\varphi_{1} \xi_{2}$ is the Reeb vector field of the contact form $\eta_{3}$. If we define the tensor $\varphi_{3}$ by

$$
\varphi_{3} \xi_{3}=0, \varphi_{3} \xi_{1}=-\varepsilon \xi_{2}, \varphi_{3} \xi_{2}=\varepsilon \xi_{1},
$$

since $\eta_{3}\left(\xi_{i}\right)=\delta_{3 i}$, we have $\varphi_{3}^{2}=-I+\eta_{3} \otimes \xi_{3}$. Consider the tensors

$$
\begin{aligned}
\eta & =-\bar{\varepsilon} \eta_{3}, \quad \varphi=\varphi_{3}, \quad \xi=-\bar{\varepsilon} \xi_{3}, \\
g_{\kappa} & =\varepsilon \bar{\varepsilon} \kappa g+(1-\varepsilon \bar{\varepsilon} \kappa) \eta_{3} \otimes \eta_{3}, \text { where we put } \bar{\varepsilon}=\operatorname{sign}(\varepsilon \kappa) .
\end{aligned}
$$

It is not difficult to see that these tensors satisfy $\varphi^{2}=-I+\eta \otimes \xi, \eta=g_{K}(\xi, \cdot)$ and $d \eta=g_{\kappa}(\cdot, \varphi)$. Therefore, $\left(\eta, \varphi, \xi, g_{\kappa}\right)$ is a contact metric structure on the three manifold $M$. Moreover, this structure is Sasakian if and only if the almost contact metric structure $(\eta, \varphi, \xi)$ is normal.

Recall that in dimension three, any almost CR structure is integrable, then by using Theorem 11 of [25] we get that the almost contact structure $(\eta, \varphi, \xi)$ is normal (equivalently, the induced almost CR structure is normal) if and only if $\xi$ is a CR Reeb vector field, that is, the tensor $\mathcal{L}_{\xi} J$ vanishes, where $J=\varphi_{\mid \operatorname{ker} \eta}, \operatorname{ker} \eta=\operatorname{span}\left(\xi_{1}, \xi_{2}\right)$. By using (7.2),

$$
\begin{aligned}
-\bar{\varepsilon}\left(\mathcal{L}_{\xi} J\right) \xi_{1} & =\left(\mathcal{L}_{\xi_{3}} \varphi_{3}\right) \xi_{1}=\left[\xi_{3}, \varphi_{3} \xi_{1}\right]-\varphi_{3}\left[\xi_{3}, \xi_{1}\right]=2(\varepsilon+1) \xi_{1},-\bar{\varepsilon}\left(\mathcal{L}_{\xi} J\right) \xi_{2}=\left(\mathcal{L}_{\xi_{3}} \varphi_{3}\right) \\
\xi_{2} & =\left[\xi_{3}, \varphi_{3} \xi_{2}\right]-\varphi_{3}\left[\xi_{3}, \xi_{2}\right]=-2(\varepsilon+1) \xi_{2} .
\end{aligned}
$$


Then, $(\xi, \eta, \varphi)$ is normal if and only if $\varepsilon+1=0$, that is, $\left(\eta_{1}, \eta_{2}\right)$ is a taut contact circle.

Next, we examine the property (3) of Theorem 7.5. Consider the contact metric structure $\left(\eta=-\bar{\varepsilon} \eta_{3}, g_{\kappa}=\varepsilon \bar{\varepsilon} \kappa g+(1-\varepsilon \bar{\varepsilon} \kappa) \eta_{3} \otimes \eta_{3}\right), \bar{\varepsilon}=\operatorname{sign}(\varepsilon \kappa)$, which is Sasakian if and only if $\varepsilon=-1$. We note that the metric $g_{\kappa}=g$ if and only if the Webster function $\kappa= \pm 1$.

Now, for $\kappa= \pm 1$, the vector fields $\left(\xi_{1}, \xi_{2}, \xi_{3}\right)$ are $g$-orthonormal and satisfy (7.2), for which $\widetilde{M}$ has a Lie group structure isomorphic to $S U(2)$ or $\widetilde{S L}(2, \mathbb{R})$; moreover,

$$
\left(\eta_{1}, \eta_{2}, g\right), \quad\left(\eta_{1},-\bar{\varepsilon} \eta_{3}, g\right), \quad\left(\eta_{2},-\bar{\varepsilon} \eta_{3}, g\right)
$$

are three left invariant bi-contact metric structures with

$$
\eta_{3} \wedge d \eta_{3}=-\varepsilon \kappa \eta_{2} \wedge d \eta_{2}=\kappa \eta_{1} \wedge d \eta_{1}, \quad \eta_{i} \wedge d \eta_{j}=0, i \neq j
$$

So, we distinguish the following cases.

a) $\quad(\kappa, \varepsilon)=(1,-1)$. In this case $\left(\eta, \xi, \varphi, g_{\kappa}\right)=\left(\eta_{3}, \xi_{3}, \varphi_{3}, g\right)$ and $\tilde{M}$ is the Lie group $S U(2)$. Moreover, the tensors $\left(\xi_{i}, \eta_{i}, \varphi_{i}\right), i=1,2,3$, related to the bi-contact metric structures (7.6), satisfy the condition (2.3). Thus, $\left(\eta_{1}, \eta_{2}, \eta_{3}, g\right)$ is a contact metric 3 -structure. In particular, by Remark 5.2, the triple $\left(\eta_{1}, \eta_{2}, \eta_{3}\right)$ defines a taut contact 2-sphere. On the other hand, a contact metric 3-structure is a Sasakian 3-structure (see, for example, [3] p.293) and $g$ is of constant sectional curvature +1 .

b) $\quad(\kappa, \varepsilon)=(-1,1),(1,1),(-1,-1)$. In this case $\widetilde{M}$ is the Lie group $\widetilde{S L}(2, \mathbb{R})$.

$\left.b_{1}\right)$ If $(\kappa, \varepsilon)=(-1,1)$, then $\left(\eta, \xi, \varphi, g_{\kappa}\right)=\left(\eta_{3}, \xi_{3}, \varphi_{3}, g\right)$ is not Sasakian and thus $\left(\eta_{1}, \eta_{2}, \eta_{3}, g\right)$ is not a contact metric 3-structure. Moreover, since $\kappa+\varepsilon=0$, from (7.4) we have that $\left(\eta_{1}, g\right)$ is Sasakian. Besides, by using (7.5) a direct computation gives that the Ricci tensor of $g$ is Ric $=-6 g+8 \eta_{1} \otimes \eta_{1}$. Then, if we consider the corresponding Lorentzian-Sasakian structure $\left(\eta_{1}, g_{L}=g-2 \eta_{1} \otimes \eta_{1}\right)$, from formula (22) of [25], the corresponding Ricci tensor is given by $R i c_{L}=R i c+4 g-4 \eta_{1} \otimes \eta_{1}=-2 g_{L}$, and thus $g_{L}$ is a Lorentzian metric of constant sectional curvature -1 .

$\left.b_{2}\right)$ If $(\kappa, \varepsilon)=(1,1)$, also in this case $\left(\eta, \xi, \varphi, g_{\kappa}\right)=\left(-\eta_{3},-\xi_{3}, \varphi_{3}, g\right)$ is not Sasakian and thus $\left(\eta_{1}, \eta_{2},-\eta_{3}, g\right)$ is not a contact metric 3-structure. Moreover, since $\kappa=\varepsilon$, from (7.4) we have that $\left(\eta_{2}, g\right)$ is Sasakian. Then, as in the case $\left.b_{1}\right)$, we get that $\left(\eta_{2}, g_{L}=g-2 \eta_{2} \otimes \eta_{2}\right)$ is a Lorentzian-Sasakian structure with $g_{L}$ Lorentzian metric of constant sectional curvature -1 .

$\left.b_{3}\right)$ If $(\kappa, \varepsilon)=(-1,-1)$, the structures $\left(\eta_{1}, g\right),\left(\eta_{2}, g\right)$ are not Sasakian, thus $\left(\eta_{1}, \eta_{2},-\eta_{3}, g\right)$ is not a contact metric 3 -structure, but the structure $\left(\eta, \xi, \varphi, g_{\kappa}\right)=\left(-\eta_{3},-\xi_{3}, \varphi_{3}, g\right)$ is Sasakian. Then, as in the case $\left.b_{1}\right)$, we get that $\left(-\eta_{3}, g_{L}=g-2 \eta_{3} \otimes \eta_{3}\right)$ is a Lorentzian-Sasakian structure with $g_{L}$ Lorentzian metric of constant sectional curvature -1 .

Finally, in all the cases $b_{i}$ ), $i=1,2,3$, from Proposition 5.1, follows that the 1-forms $\eta_{1}, \eta_{2}, \eta_{3}$ define a taut contact 2-hyperboloid.

Summing up, we get

Corollary 7.6 Let $\left(\eta_{1}, \eta_{2}, g\right)$ be a bi-contact metric structure on the three-manifold $M$ with the Webster function $\kappa \neq 0$ everywhere. Then, the metric $g_{\kappa}=g$ if and only if $\kappa=$ const. $= \pm 1$. 
In this case, i.e., for $\kappa=$ const. $= \pm 1$,

$$
\left(\eta_{1}, \eta_{2}, g\right),\left(\eta_{1},-\kappa \varepsilon \eta_{3}, g\right) \text { and }\left(\eta_{2},-\kappa \varepsilon \eta_{3}, g\right)
$$

are three left invariant bi-contact metric structures and $\widetilde{M}$ is either $S U(2)$ or $\widetilde{S L}(2, \mathbb{R})$.

More precisely, we have the following.

- If $(\kappa, \varepsilon)=(1,-1), \widetilde{M}$ is $S U(2)$ and $\left(\eta_{1}, \eta_{2}, \eta_{3}, g\right)$ is a 3-Sasakian structure on it, where $\left(\eta_{1}, \eta_{2}, \eta_{3}\right)$ is a taut contact 2 -sphere and $g$ is of constant sectional curvature +1 .

- If $(\kappa, \varepsilon)=(-1,1),(1,1),(-1,-1), \widetilde{M}$ is $\widetilde{S L}(2, \mathbb{R})$ and the 1 -forms $\eta_{1}, \eta_{2}, \eta_{3}$ define a taut contact 2-hyperboloid. Besides, for $(\kappa, \varepsilon)=(-1,-1)($ resp. $(\kappa, \varepsilon)=(-1,1),(1,1))$ the structure $\left(-\eta_{3}, g_{L}=g-2 \eta_{3} \otimes \eta_{3}\right) \quad\left(\right.$ resp. $\quad\left(\eta_{1}, g_{L}=g-2 \eta_{1} \otimes \eta_{1}\right)$, $\left.\left(\eta_{2}, g_{L}=g-2 \eta_{2} \otimes \eta_{2}\right)\right)$ is a Lorentzian-Sasakian structure with $g_{L}$ Lorentzian metric of constant sectional curvature -1 .

Remark 7.7 From Corollary 7.6 we get that the Lie group $\widetilde{S L}(2, \mathbb{R})$ admits three left invariant bi-contact metric structures, with the same associated metric, which do not define a contact metric 3-structure.

\section{Generalized Finsler structures and bi-contact metric structures}

The main purpose of this section is to see how the taut contact hyperbolas are related to generalized Finsler structures, and construct examples of bi-contact metric structures $\left(\eta_{1}, \eta_{2}, g\right)$ with $\left(\eta_{1}, \eta_{2}\right)$ taut contact hyperbola. On the other hand, in [24], we posed the question to find examples (if there exist) of bi-contact metric structures $\left(\eta_{1}, \eta_{2}, g\right)$ on 3 -manifolds which are not homogeneous, and thus with the Webster function $\kappa$ non-constant, where the 1-forms $\left(\eta_{1}, \eta_{2}\right)$ satisfy the conditions that define a taut contact hyperbola. So, by this study we give, in particular, a positive answer to this question (see Example 8.2).

Let $M$ be a three-manifold. Following R. Bryant [5, 6], a coframe $\left(\omega_{1}, \omega_{2}, \omega_{3}\right)$ on $M$ is said to be a $(I, J, K)$-generalized Finsler structure if it satisfies the following structure equations

$$
\left\{\begin{array}{c}
d \omega_{1}=-\omega_{2} \wedge \omega_{3}, \\
d \omega_{2}=\omega_{1} \wedge \omega_{3}+I \omega_{3} \wedge \omega_{2}, \\
d \omega_{3}=-K \omega_{1} \wedge \omega_{2}-J \omega_{2} \wedge \omega_{3},
\end{array}\right.
$$

where $(I, J, K)$ are smooth functions on $M$, known as the main scalar, the Landsberg curvature and the flag curvature, respectively.

We note that if $\left(\omega_{1}, \omega_{2}, \omega_{3}\right)$ is a $(I, J, K)$-generalized Finsler structure, then $\left(\omega_{1},-\omega_{2},-\omega_{3}\right)$ is a $(-I,-J, K)$-generalized Finsler structure. As remarked in [5] and [6], the difference between the notions of Finsler structure and generalized Finsler structure is global in nature, that is, any generalized Finsler structure is locally diffeomorphic to a Finsler structure, hence $M$ can be realized locally as the unit sphere bundle of a Finsler surface $(N, F)$ in such a way that the given coframing is the canonical coframing induced on $M$ by the (local) Finsler structure $F$. 
In the sequel, for a smooth function $f$ on $M$ equipped with a generalized Finsler structure, we put $d f=\sum_{i=1}^{3} f_{i} \omega_{i}$. Computing the exterior derivative of the structure equations (8.1), one gets the so called Bianchi identities (cf. [5], Section 1; [6], Section 2.2)

$$
I_{1}=J, \quad J_{1}=-K_{3}-K I .
$$

In particular, $I=$ const. implies $J=0 ; \quad J=$ const. and $K=$ const. $\neq 0$ imply $I=J=0$. When $I=J=0$, the generalized Finsler structure is locally a Riemannian structure.

Denote by $\Omega$ the volume form $\omega_{1} \wedge \omega_{2} \wedge \omega_{3}$. From (8.1), a simply computation gives

$$
\begin{aligned}
& \omega_{2} \wedge d \omega_{2}=\omega_{1} \wedge d \omega_{1}=-\Omega, \quad \omega_{3} \wedge d \omega_{3}=K \omega_{1} \wedge d \omega_{1}=-K \Omega, \quad \omega_{1} \wedge d \omega_{2}=-I \Omega, \\
& \omega_{2} \wedge d \omega_{1}=0, \quad \omega_{1} \wedge d \omega_{3}=-J \Omega, \quad \omega_{3} \wedge d \omega_{1}=0, \quad \omega_{2} \wedge d \omega_{3}=\omega_{3} \wedge d \omega_{2}=0 .
\end{aligned}
$$

Then, from Theorem 7.1, we get

Proposition 8.1 Let $\left(\omega_{1}, \omega_{2}, \omega_{3}\right)$ be a $(I, J, K)$-generalized Finsler structure on a threemanifold $M$. Then

a) $\left(\omega_{1}, \omega_{2}\right)$ defines a bi-contact metric structure if and only if $\varepsilon=-1, I=J=0$.

b) $\left(\omega_{1}, \omega_{3}\right)$ defines a bi-contact metric structure if and only if $K=-\varepsilon, J=I=0$.

c) $\left(\omega_{2}, \omega_{3}\right)$ defines a bi-contact metric structure if and only if $K=-\varepsilon$.

Bryant et al. [6] studied Finsler surfaces of constant flag curvature $K=0$, \pm 1 , with a Killing field.

Next, we discuss separately the cases $a$ ), b), c).

- The case a) : $\varepsilon=-1, I=J=0$. In this case the triple of 1 -forms $\left(\eta_{1}, \eta_{2}, \eta_{3}\right)=(1 / 2)\left(\omega_{1}, \omega_{2}, \omega_{3}\right)$ satisfies (III) of Theorem 7.1 , where $\left(\eta_{1}, \eta_{2}\right)$ is a taut contact circle with the Webster function $\kappa=K$. So, if the flag curvature $K$ is $\neq 0$ everywhere, from Theorem 7.5 follows that $\omega_{3}$ is a Killing contact form. Moreover, in this case, we have a generalized Riemann structure in the sense of [7]. Of course, a bi-contact metric structure with $\varepsilon=-1$ defines a $(0,0, \kappa)$ generalized Finsler structure.

A model for this type of structure is implicitly given in [24]. More precisely, consider the space $\mathbb{R}^{3}\left(x_{1}, x_{2}, t\right)$, a smooth function $\sigma=\sigma\left(x_{1}, x_{2}\right)$ and put $\sigma_{1}=\partial \sigma / \partial x_{1}$, $\sigma_{2}=\partial \sigma / \partial x_{2}, \sigma_{11}=\partial^{2} \sigma / \partial x_{1}^{2}$ and $\sigma_{22}=\partial^{2} \sigma / \partial x_{2}^{2}$. Then, the 1-forms

$$
\begin{aligned}
& \omega_{1}=e^{\sigma}\left((\cos t) d x_{1}+(\sin t) d x_{2}\right), \\
& \omega_{2}=e^{\sigma}\left(-(\sin t) d x_{1}+(\cos t) d x_{2}\right), \\
& \omega_{3}=-\sigma_{2} d x_{1}+\sigma_{1} d x_{2}+d t,
\end{aligned}
$$

define a coframe on $\mathbb{R}^{3}\left(x_{1}, x_{2}, t\right)$. Moreover, they satisfy the structure equations (8.1) of a generalized Finsler structure with $I=J=0$ and $K=-e^{-2 \sigma}\left(\sigma_{11}+\sigma_{22}\right)$. We note that if $(N, G)$ is a Riemannian surface, using isothermal local coordinates $\left(x_{1}, x_{2}\right)$ on $N$, the Riemannian metric $G$ is given by $G=e^{2 \sigma}\left(d x_{1}^{2}+d x_{2}^{2}\right)$ and, in terms of these coordinates, the function $K=-e^{-2 \sigma}\left(\sigma_{11}+\sigma_{22}\right)$ is its Gaussian curvature.

- In the case $b): K=-\varepsilon, I=J=0$, the triple of 1 -forms $\left(\eta_{1}, \eta_{2}, \eta_{3}\right)=(1 / 2)\left(\omega_{1}, \omega_{3},-\omega_{2}\right)$ satisfies $(I I I)$ of Theorem 7.1 with $\kappa=1$, where $\left(\eta_{1}, \eta_{2}\right)$ is a taut contact hyperbola (resp. circle) if $\varepsilon=1$ (resp. $\varepsilon=-1$ ). 
- In the case $c$ ): $K=-\varepsilon$, the most interesting case for our study, the structure equations become

$$
\left\{\begin{array}{c}
d \omega_{1}=-\omega_{2} \wedge \omega_{3} \\
d \omega_{2}=\omega_{1} \wedge \omega_{3}+I \omega_{3} \wedge \omega_{2} \\
d \omega_{3}=\varepsilon \omega_{1} \wedge \omega_{2}-J \omega_{2} \wedge \omega_{3}
\end{array}\right.
$$

Then, the 1-forms

$$
\eta_{1}=(1 / 2) \omega_{3}, \quad \eta_{2}=(1 / 2) \omega_{2} \text { and } \eta_{3}=(1 / 2)\left(\varepsilon \omega_{1}+J \omega_{3}-\varepsilon I \omega_{2}\right)
$$

satisfy:

$$
\begin{aligned}
d \eta_{1} & =(1 / 2) d \omega_{3}=(1 / 2)\left(\varepsilon \omega_{1} \wedge \omega_{2}-J \omega_{2} \wedge \omega_{3}\right)=2 \eta_{3} \wedge \eta_{2}, \\
-2 \varepsilon \eta_{1} \wedge \eta_{3} & =-(1 / 2) \omega_{3} \wedge\left(\omega_{1}+J \varepsilon \omega_{3}-I \omega_{2}\right)=(1 / 2) d \omega_{2}=d \eta_{2} .
\end{aligned}
$$

Besides,

$$
\begin{aligned}
2 d \eta_{3} & =\left(\varepsilon d \omega_{1}+d J \wedge \omega_{3}+J d \omega_{3}-\varepsilon d I \wedge \omega_{2}-\varepsilon I d \omega_{2}\right) \\
& =\left(J_{2}-J^{2}+\varepsilon I_{3}+\varepsilon I^{2}-\varepsilon\right) \omega_{2} \wedge \omega_{3}+\left(J_{1}-\varepsilon I\right) \omega_{1} \wedge \omega_{3}+\left(\varepsilon J-\varepsilon I_{1}\right) \omega_{1} \wedge \omega_{2},
\end{aligned}
$$

and thus, by using (8.2), we have

$$
d \eta_{3}=2 \kappa \eta_{2} \wedge \eta_{1} \text {, where } \kappa=\left(J_{2}-J^{2}+\varepsilon I_{3}+\varepsilon I^{2}-\varepsilon\right) .
$$

Therefore, for $K=-1$ (resp. $K=1$ ), we get taut contact hyperbolas (resp. circles) with the Webster function $\kappa$, in general, non-constant. If $K=1$ and $\kappa \neq 0$ everywhere, from Theorem 7.5 follows that $\eta_{3}$ is a Killing contact form.

Next, we give an explicit example of bi-contact metric structure $\left(\eta_{1}, \eta_{2}, g\right)$ where $\left(\eta_{1}, \eta_{2}\right)$ is a taut contact hyperbola with the Webster function $\kappa$ non-constant.

Example 8.2 Let $M$ be a connected open subset of $\mathbb{R}^{3}$. On $M$ we consider the following 1-forms

$$
\omega_{1}=d x+x d y+d z, \quad \omega_{2}=-\frac{\cosh z}{f(x)} d x+f(x)(\sinh z) d y, \quad \omega_{3}=-\frac{\sinh z}{f(x)} d x+f(x)(\cosh z) d y
$$

where $f(x)$ is a positive smooth function defined on $M$. These forms satisfy:

$$
\begin{aligned}
d \omega_{1} & =d x \wedge d y=\omega_{3} \wedge \omega_{2}, \\
d \omega_{2} & =-\frac{\sinh z}{f(x)} d z \wedge d x+f^{\prime}(x)(\sinh z) d x \wedge d y+f(x)(\cosh z) d z \wedge d y \\
d \omega_{3} & =-\frac{\cosh z}{f(x)} d z \wedge d x+f^{\prime}(x)(\cosh z) d x \wedge d y+f(x)(\sinh z) d z \wedge d y, \\
\omega_{1} \wedge \omega_{3} & =-\frac{\sinh z}{f(x)} d z \wedge d x+f(x) \cosh z(d z \wedge d y)+\left(f(x)(\cosh z)+x \frac{\sinh z}{f(x)}\right) d x \wedge d y, \\
\omega_{1} \wedge \omega_{2} & =-\frac{\cosh z}{f(x)} d z \wedge d x+f(x)(\sinh z) d z \wedge d y+\left(f(x)(\sinh z)+x \frac{\cosh z}{f(x)}\right) d x \wedge d y .
\end{aligned}
$$

So, one gets 


$$
d \omega_{1}=\omega_{3} \wedge \omega_{2}, \quad d \omega_{2}=\omega_{1} \wedge \omega_{3}+I \omega_{3} \wedge \omega_{2}, \quad d \omega_{3}=\omega_{1} \wedge \omega_{2}-J \omega_{2} \wedge \omega_{3},
$$

where

$$
I=\left(f^{\prime}-(x / f)\right) \sinh z-f \cosh z \text { and } J=\left(f^{\prime}-(x / f)\right) \cosh z-f \sinh z .
$$

Therefore, $\left(\omega_{1}, \omega_{2}, \omega_{3}\right)$ is a $(I, J,-1)$ generalized Finsler structure on $M$. Then, by the discussion of the case $c$ ) of Proposition $8.1,\left(\eta_{1}, \eta_{2}\right)=(1 / 2)\left(\omega_{3}, \omega_{2}\right)$ is a taut contact hyperbola. In this case, the 1 -form $\eta_{3}$ is given by

$$
\eta_{3}=(1 / 2)\left(\omega_{1}+J \omega_{3}-I \omega_{2}\right)=\ldots=(1 / 2)\left(d z+f^{\prime}(x) f(x) d y\right) .
$$

Since $2 d \eta_{3}=\left(f^{\prime} f\right)^{\prime}(x) d x \wedge d y=\left(f^{\prime} f\right)^{\prime}(x) \omega_{3} \wedge \omega_{2}=-4\left(f^{\prime} f\right)^{\prime}(x) \eta_{2} \wedge \eta_{1}$, we get

$$
d \eta_{3}=2 \kappa \eta_{2} \wedge \eta_{1}
$$

where the Webster $\kappa$ function is given by

$$
\kappa(x)=-\left(f^{\prime} f\right)^{\prime}(x)=-(1 / 2)\left(f^{2}\right)^{\prime \prime}(x) .
$$

The frame $\left(\xi_{1}, \xi_{2}, \xi_{3}\right)$, dual of the coframe $\left(\eta_{1}, \eta_{2}, \eta_{3}\right)$, is given by

$$
\begin{aligned}
& \xi_{1}=2\left(f(x)(\sinh z) \partial_{x}+\frac{\cosh z}{f(x)} \partial_{y}-f^{\prime}(x)(\cosh z) \partial_{z}\right), \\
& \xi_{2}=-2\left(f(x)(\cosh z) \partial_{x}+\frac{\sinh z}{f(x)} \partial_{y}-f^{\prime}(x)(\sinh z) \partial_{z}\right), \quad \xi_{3}=2 \partial_{z},
\end{aligned}
$$

where $\xi_{1}, \xi_{2}$ are the Reeb vector fields of $\eta_{1}, \eta_{2}$.

Then, if $g$ is the Riemannian metric defined by $g\left(\xi_{i}, \xi_{j}\right)=\delta_{i j},\left(\eta_{1}, \eta_{2}, g\right)$ is a bi-contact metric structure with the Webster scalar curvatures

$$
\mathcal{W}_{1}=-\left(\left(f^{2}\right)^{\prime \prime}(x)+2\right) / 4 \quad \text { and } \quad \mathcal{W}_{2}=\left(\left(f^{2}\right)^{\prime \prime}(x)-2\right) / 4
$$

In this example, we have a family of $\left(I_{f}, J_{f},-1\right)$ generalized Finsler structures, depending on a function $f(x)$, that define taut contact hyperbolas. In particular, this family contains generalized Finsler structures that define left invariant taut contact hyperbolas on the Lie groups $S o l^{3}$ and $\widetilde{S L}(2, R)$. More precisely,

- Consider $M=\mathbb{R}^{3}$ and the $\left(I_{f}, J_{f},-1\right)$ generalized Finsler structure corresponding to the function $f(x)=1$. Then, the 1 -forms $\left(\eta_{1}, \eta_{2}\right)=(1 / 2)\left(\omega_{3}, \omega_{2}\right)$ and $\eta_{3}$ are given by

$$
\eta_{1}=(-(\sinh z) d x+(\cosh z) d y) / 2, \quad \eta_{2}=(-(\cosh z) d x+(\sinh z) d y) / 2, \quad \eta_{3}=(1 / 2) d z,
$$

and the dual vector fields

$$
\begin{aligned}
& \xi_{1}=2\left((\sinh z) \partial_{x}+(\cosh z) \partial_{y}\right), \quad \xi_{2}=-2\left((\cosh z) \partial_{x}+(\sinh z) \partial_{y}\right), \\
& \xi_{3}=2 \partial_{z}
\end{aligned}
$$

satisfy (3.7), and hence define on $\mathbb{R}^{3}$ a Lie group structure isomorphic to $\mathrm{Sol}^{3}$.

- Consider $\quad M=\mathbb{R}_{+}^{3}=\left\{(x, y, z) \in \mathbb{R}^{3}: x>0\right\}$ and the $\left(-1, I_{f}, J_{f}\right)$ generalized Finsler structure corresponding to the function $f(x)=x$. Then, the 1 -forms $\left(\eta_{1}, \eta_{2}\right)=(1 / 2)\left(\omega_{3}, \omega_{2}\right)$ and $\eta_{3}$ are given by 


$$
\begin{aligned}
& \eta_{1}=\left(-\frac{\sinh z}{x} d x+x(\cosh z) d y\right) / 2, \quad \eta_{2}=\left(-\frac{\cosh z}{x} d x+x(\sinh z) d y\right) / 2, \\
& \eta_{3}=(1 / 2)(x d y+d z)
\end{aligned}
$$

and the dual vector fields

$$
\begin{aligned}
& \xi_{1}=2\left(x(\sinh z) \partial_{x}+\frac{\cosh z}{x} \partial_{y}-(\cosh z) \partial_{z}\right), \quad \xi_{2}=-2\left(x(\cosh z) \partial_{x}+\frac{\sinh z}{x} \partial_{y}-(\sinh z) \partial_{z}\right), \\
& \xi_{3}=2 \partial_{z},
\end{aligned}
$$

satisfy (3.8), and hence define on $\mathbb{R}_{+}^{3}$ a Lie group structure isomorphic to $\widetilde{S L}(2, \mathbb{R})$.

Remark 8.3 About the Webster curvature, we recall that every contact structure on a compact orientable three-manifold has a contact form and an associated Riemannian metric whose Webster scalar curvature is either a constant $\leq 0$ or is everywhere strictly positive (see the main result of [9]). On the other hand, every compact orientable three-manifold $\mathrm{M}$ has a contact structure [19]. Therefore, every compact orientable three-manifold $M$ has a contact Riemannian structure whose Webster scalar curvature is either a constant $\leq 0$ or is everywhere strictly positive.

Now, from (8.3), it is not difficult to find a positive function $f(x)$ for which $\mathcal{W}_{1}$ be a strictly negative function and $\mathcal{W}_{2}$ be a strictly positive function. On the other hand, on a three-manifold, a contact Riemannian structure determines a non-degenerate CR structure with the same Webster scalar curvature (cf., for example, [25] p.30). So, we get the following

Proposition 8.4 Any connected open subset of $\mathbb{R}^{3}$ admits a non-degenerate CR structure whose Webster scalar curvature is a strictly negative function and a non-degenerate $C R$ structure whose Webster scalar curvature is a strictly positive function.

\section{Final remark}

Of course, it is an open question to give a classification of three-manifolds which admit a taut contact hyperbola. Recall that the homothety class of a taut contact circle is defined by multiplication by the same positive function and by a rotation of constant angle [13]. Similarly, we can define the homothety class of a taut contact hyperbola. If $f$ is a positive smooth function and $\left(\eta_{1}, \eta_{2}\right)$ a pair of contact forms on a three-manifold $M$, the contact forms $\left(\tilde{\eta}_{1}=f \eta_{1}, \tilde{\eta}_{2}=f \eta_{2}\right)$ satisfy

$$
\tilde{\eta}_{i} \wedge d \tilde{\eta}_{i}=f^{2} \eta_{i} \wedge d \eta_{i},(i=1,2), \quad \tilde{\eta}_{1} \wedge d \tilde{\eta}_{2}+\tilde{\eta}_{2} \wedge d \tilde{\eta}_{1}=f^{2}\left(\eta_{1} \wedge d \eta_{2}+\eta_{2} \wedge d \eta_{1}\right) .
$$

Then,

- $\quad\left(\eta_{1}, \eta_{2}\right)$ is a taut contact hyperbola if and only if $\left(\tilde{\eta}_{1}, \tilde{\eta}_{2}\right)$ is a taut contact hyperbola.

Moreover, if $\left(\eta_{1}, \eta_{2}\right)$ is a taut contact hyperbola and $\left(\eta_{1}^{\prime}, \eta_{2}^{\prime}\right)$ is obtained from $\left(\eta_{1}, \eta_{2}\right)$ by a hyperbolic rotations of constant angle, it is not difficult to see that

- $\quad\left(\eta_{1}, \eta_{2}\right)$ is a taut contact hyperbola if and only if $\left(\eta_{1}^{\prime}, \eta_{2}^{\prime}\right)$ is a taut contact hyperbola.

This suggests to define the homothety class of a taut contact hyperbola by multiplication by the same positive function and by a hyperbolic rotation of constant angle. Hence, to classify taut contact hyperbolas is equivalent to classify their homothety classes. 
Funding Open access funding provided by Università del Salento within the CRUI-CARE Agreement.

Open Access This article is licensed under a Creative Commons Attribution 4.0 International License, which permits use, sharing, adaptation, distribution and reproduction in any medium or format, as long as you give appropriate credit to the original author(s) and the source, provide a link to the Creative Commons licence, and indicate if changes were made. The images or other third party material in this article are included in the article's Creative Commons licence, unless indicated otherwise in a credit line to the material. If material is not included in the article's Creative Commons licence and your intended use is not permitted by statutory regulation or exceeds the permitted use, you will need to obtain permission directly from the copyright holder. To view a copy of this licence, visit http://creativecommons.org/licenses/by/4.0/.

\section{References}

1. Abbassi, K.M.T., Calvaruso, G.: g-natural contact metrics on unit tangent sphere bundles. Monatsh. Math. 151, 189-209 (2006)

2. Bande, G., Kotschick, D.: The geometry of symplectic pairs. Trans. Am. Math. Soc. 358(4), 16431655 (2005)

3. Blair, D.E.: Riemannian Geometry of Contact and Symplectic Mani-folds, 2nd edn. Birkhäuser, Boston, Basel, Berlin (2010)

4. Boyer, C.P., Galicki K.: Sasakian Geometry, Oxford Mathematical Monographs. Oxford University Press, Oxford (2008)

5. Bryant, R.: Finsler structures on the 2-sphere satisfying $K=1$. Contemp. Math. 196, $27-41$ (1996)

6. Bryant, R.L., Huang, L., Mo, X.: On Finsler surfaces of constant flag curvature with a killing field. J. Geom. Phys. 116, 345-357 (2017)

7. Bryant, R.: Finsler surfaces with prescribed curvature conditions, preprint, pp. 99 (1995)

8. Catoni, F., Cannata, R., Catoni, V., Zampetti, P.: Hyperbolic trigonometry in two-dimensional spacetime geometry. Nuovo Cimento Soc. Ital. Fis. B 118(5), 475-492 (2003)

9. Chern, S.S., Hamilton, R.S.: On Riemannian Metrics Adapted to Three-dimensional Contact Manifolds Lect Notes in Math 1111, pp. 279-305. Springer-Verlag, Berlin, New-York (1985)

10. Dragomir, S., Perrone D.: Harmonic Vector Fields: Variational Principles and Differential Geometry. Elsevier Science Ltd, Amsterdam (2011)

11. Eliashberg, Y.: Classification of overtwisted contact structures on 3-manifolds. Invent. Math. 98(3), 623-637 (1989)

12. Eliashberg, Y. M., Thurston, W. P.: Confoliations. University Lecture Series, 13, Amer. Math. Soc., (1998)

13. Geiges, H., Gonzalo, J.: Contact geometry and complex surfaces. Invent. Math. 121, 147-209 (1995)

14. Geiges, H.: Symplectic couples on 4-manifolds. Duke Math. J. 85, 701-711 (1996)

15. Geiges, H., Gonzalo, J.: Contact circles on 3-manifolds. J. Differ. Geom. 46, 236-286 (1997)

16. Geiges, H., Gonzalo, J.: Contact spheres and hyperkaehler geometry. Commun. Math. Phys. 287, 719748 (2009)

17. Geiges, H., Gonzalo, J.: Fifteen years of contact circles and contact spheres. Acta Math. Vietnam 38, 145-164 (2013)

18. Hozoori, S.: Dynamics and topology of conformally Anosov contact 3-manifolds. Differ. Geom. Appl. 73, 101679 (2020)

19. Martinet, J.: Formes de contact sur les varietes de dimension 3. Proc. Liverpool Singularities Symp II, Springer Lecture Notes in Math, 209, 142-163 (1971)

20. Milnor, J.: Curvature of left invariant metrics on Lie groups. Adv. Math. 21, 293-329 (1976)

21. Mitsumatsu, Y.: Anosov flows and non-Stein symplectic manifolds. Ann. Inst. Fourier 45, 1407-1421 (1995)

22. Perrone, D.: Homogeneous contact Riemannian three-manifolds. Illinois Math. 42(2), 243-256 (1998)

23. Perrone, D.: Contact metric manifolds whose characteristic vector field is a harmonic vector field. Diff. Geom. Appl. 20, 367-378 (2004)

24. Perrone, D.: Taut contact circles and bi-contact metric structures on three-manifolds. Ann. Global Anal. Geom. 52, 213-235 (2017)

25. Perrone, D.: Contact semi-Riemannian structures in CR geometry some aspects. Special issue applications of differential geometry. Axioms 8(1), 6 (2019) 
26. Tanno, S.: Variational problems on contact Riemannian manifolds. Trans. Am. Math. Soc. 314(1), 349-379 (1989)

27. Zessin, M.: On contact p-spheres. Ann. Inst. Fourier. 55(4), 1167-1194 (2005)

Publisher's Note Springer Nature remains neutral with regard to jurisdictional claims in published maps and institutional affiliations. 